This is a self-archived version of an original article. This version may differ from the original in pagination and typographic details.

Author(s): Koskela, Pekka; Xiao, Jie; Zhang, Yi Ru-Ya; Zhou, Yuan

Title: A quasiconformal composition problem for the Q-spaces

Year: 2017

Version: Accepted version (Final draft)

Copyright: ㄷ 2019 EMS Publishing House

Rights: In Copyright

Rights url: http://rightsstatements.org/page//nC/1.0/?language=en

Please cite the original version:

Koskela, P., Xiao, J., Zhang, Y. R.-Y., \& Zhou, Y. (2017). A quasiconformal composition problem for the Q-spaces. Journal of the European Mathematical Society, 19(4), 1159-1187.

https://doi.org/10.4171/JEMS/690 


\title{
A QUASICONFORMAL COMPOSITION PROBLEM FOR THE $Q$-SPACES
}

\author{
PEKKA KOSKELA, JIE XIAO, YI RU-YA ZHANG AND YUAN ZHOU
}

\begin{abstract}
Aвstract. Given a quasiconformal mapping $f: \mathbb{R}^{n} \rightarrow \mathbb{R}^{n}$ with $n \geq 2$, we show that (un-)boundedness of the composition operator $\mathbf{C}_{f}$ on the spaces $Q_{\alpha}\left(\mathbb{R}^{n}\right)$ depends on the index $\alpha$ and the degeneracy set of the Jacobian $J_{f}$. We establish sharp results in terms of the index $\alpha$ and the local/global self-similar Minkowski dimension of the degeneracy set of $J_{f}$. This gives a solution to [3, Problem 8.4] and also reveals a completely new phenomenon, which is totally different from the known results for Sobolev, BMO, Triebel-Lizorkin and Besov spaces. Consequently, Tukia-Väisälä's quasiconformal extension $f: \mathbb{R}^{n} \rightarrow \mathbb{R}^{n}$ of an arbitrary quasisymmetric mapping $g: \mathbb{R}^{n-p} \rightarrow \mathbb{R}^{n-p}$ is shown to preserve $Q_{\alpha}\left(\mathbb{R}^{n}\right)$ for any $(\alpha, p) \in(0,1) \times[2, n) \cup(0,1 / 2) \times\{1\}$. Moreover, $Q_{\alpha}\left(\mathbb{R}^{n}\right)$ is shown to be invariant under inversions for all $0<\alpha<1$.
\end{abstract}

\section{CONTENTS}

1. Introduction

2. Local and global Minkowski dimensions 5

3. A characterization of $Q$-spaces 9

4. Proof of Theorem 1.3 12

5. Proofs of Corollaries 1.4 and $1.5 \quad 17$

6. Proofs of Theorems 1.6 and $1.7 \quad 20$

References $\quad 26$

\section{INTRODUCTION}

Quasiconformal mappings can be characterized via invariant function spaces. For example, a homeomorphism $f: \mathbb{R}^{n} \rightarrow \mathbb{R}^{n}, n \geq 2$, is quasiconformal if and only if the composition operator $\mathbf{C}_{f}$ (given by $\mathbf{C}_{f}(u)=u \circ f$ ) is bounded on the homogeneous Sobolev space $\dot{W}^{1, n}\left(\mathbb{R}^{n}\right)$; see for example [5]. The composition property is easiest seen from the usual analytic definition, according to which a homeomorphism $f: \mathbb{R}^{n} \rightarrow \mathbb{R}^{n}, n \geq 2$, is quasiconformal if $f \in W_{\text {loc }}^{1,1}\left(\mathbb{R}^{n} ; \mathbb{R}^{n}\right)$ and there is a constant $K \geq 1$ so that

$$
|D f(x)|^{n} \leq K J_{f}(x), \quad \text { a.e. } x \in \mathbb{R}^{n} .
$$

2000 Mathematics Subject Classification. 42B35; 46E30; 47B38; $30 \mathrm{H} 25$.

Key words and phrases. Quasiconformal mappings; Compositions; Q-spaces.

Pekka Koskela was supported by the Academy of Finland grant 120972; Jie Xiao was supported by NSERC of Canada (\# 202979463102000) and URP of Memorial University (\# 208227463102000); Yi Zhang and Yuan Zhou were supported by the New Teachers' Fund for Doctor Stations (\# 20121102120031) and Program for New Century Excellent Talents in University (\# NCET-11-0782) of Ministry of Education of China, and National Natural Science Foundation of China (\# 11201015). 
Indeed, modulo technicalities, one simply uses the chain rule and a change of variables. It is far less obvious that also the invariance of the Triebel-Lizorkin spaces $\dot{F}_{n / s, q}^{s}\left(\mathbb{R}^{n}\right)$ with $0<s<1$ and $n /(n+s)<q<\infty$ characterizes quasiconformality, see $[10,2,6,4]$. The difficulty here is that one has to deal with "fractional derivatives" and thus the inequality from the analytic definition is not immediately helpful. For the off-diagonal Besov spaces $\dot{B}_{n / s, q}^{s}\left(\mathbb{R}^{n}\right)$ with $q \neq n / s$, the situation is different: each homeomorphism $f$ for which $\mathbf{C}_{f}$ is bounded on $\dot{B}_{n / s, q}^{s}\left(\mathbb{R}^{n}\right)$ has to be quasiconformal and even bi-Lipschitz; these spaces are clearly bi-Lipschitz invariant, see [4]. Recall here that $f$ is bi-Lipschitz if there exists a constant $L \geq 1$ such that

$$
\frac{1}{L}|x-y| \leq|f(x)-f(y)| \leq L|x-y|, \quad \forall x, y \in \mathbb{R}^{n} .
$$

Furthermore, the John-Nirenberg space $B M O\left(\mathbb{R}^{n}\right)$ is invariant under quasiconformal mappings and each sufficiently regular homeomorphism $f$ for which $\mathbf{C}_{f}$ is a bounded operator on $B M O\left(\mathbb{R}^{n}\right)$ is necessarily quasiconformal; see $[7,1]$.

In their 2000 paper [3], Essen, Jasson, Peng and Xiao introduced the so-called Q-spaces $Q_{\alpha}\left(\mathbb{R}^{n}\right)$, $0<\alpha<1$, that satisfy

$$
\dot{W}^{1, n}\left(\mathbb{R}^{n}\right) \subset \dot{F}_{n / \alpha, n / \alpha}^{\alpha}\left(\mathbb{R}^{n}\right) \subseteq Q_{\alpha}\left(\mathbb{R}^{n}\right) \subseteq B M O\left(\mathbb{R}^{n}\right) .
$$

Each $Q_{\alpha}\left(\mathbb{R}^{n}\right)$ consists of all $u \in L_{\text {loc }}^{2}\left(\mathbb{R}^{n}\right)$ with

$$
\|u\|_{Q_{\alpha}\left(\mathbb{R}^{n}\right)}=\sup _{x_{0} \in \mathbb{R}^{n}, r>0}\left(r^{2 \alpha-n} \int_{B\left(x_{0}, r\right)} \int_{B\left(x_{0}, r\right)} \frac{|u(x)-u(y)|^{2}}{|x-y|^{n+2 \alpha}} d x d y\right)^{1 / 2}<\infty .
$$

The above definition actually makes perfect sense for all $-\infty<\alpha<\infty$, but the case $\alpha \geq 1$ (when $n \geq 2$ ) reduces to constant functions and the case $\alpha<0$ to $B M O\left(\mathbb{R}^{n}\right)$; see [3]. These spaces have received considerable interest. In [3], five open problems related to the spaces $Q_{\alpha}\left(\mathbb{R}^{n}\right)$ were posed. All but the following one of them have by now been solved.

A quasiconformal composition problem for the $Q$-spaces ([3, Problem 8.4]): Let $f$ be a quasiconformal mapping. Prove or disprove the boundedness of the composition operator $\mathbf{C}_{f}$ on $Q_{\alpha}\left(\mathbb{R}^{n}\right)$ with $\alpha \in(0,1)$.

By the above string of inclusions of function spaces, all of which except for the $Q$-spaces are known to be quasiconformally invariant, suggests that the answer should be in the positive.

We show that, surprisingly, the answer to the above question depends on the quasiconformal mapping in question through the shrinking properties of the mapping. For example, the quasiconformal mapping $f(x)=x|x|$ induces a bounded composition operator for all $0<\alpha<1$, but if the Jacobian of a quasiconformal mapping decays to zero when we approach a sufficiently large set, then the invariance may fail. Thus, the case of $Q$-spaces is be very different from the other function spaces that we discussed above.

In order to state our results, we need to introduce some terminology whose analogues have appeared in estimating the upper box-counting dimension of the singular set of a suitable weak solution of the Navier-Stokes system [8].

Definition 1.1. For a set $E \subseteq \mathbb{R}^{n}$ and every $r>0$, denote by $N_{\text {cov }}(r, E)$ the minimal number of cubes with edge length $r$ required to cover $E$. 
(i) The local self-similar Minkowski dimension of $E$ is defined as

$$
\overline{\operatorname{dim}}_{L} E=\liminf _{N \rightarrow \infty} \limsup _{r \rightarrow 0} \sup _{\substack{B \subset \mathbb{R}^{n} \\ N r \leq r_{B} \leq 1}} \frac{\log N_{\mathrm{cov}}(r, E \cap B)}{\log \left(r_{B} / r\right)},
$$

where the supremum is taken over all balls $B=B\left(x_{B}, r_{B}\right) \subset \mathbb{R}^{n}$ with $r_{B} \in[N r, 1]$.

(ii) The global self-similar Minkowski dimension of $E$ is defined as

$$
\overline{\operatorname{dim}}_{L G} E=\liminf _{N \rightarrow \infty} \sup _{\substack{r>0 \\ \sup _{B \subset \mathbb{R}^{n}}}} \frac{\log N_{\text {cov }}(r, E \cap B)}{\log \left(r_{B} / r\right)},
$$

where the first supremum is taken over all $r \in(0, \infty)$ and the second is over all balls $B=B\left(x_{B}, r_{B}\right) \subset$ $\mathbb{R}^{n}$ with $r_{B} \in[N r, \infty)$.

We also need the concept of the local Muckenhoupt class.

Definition 1.2. For a closed set $E \subseteq \mathbb{R}^{n}$ and a nonnegative function $w: \mathbb{R}^{n} \rightarrow \mathbb{R}$, we say that $w$ belongs to the local Muckenhoupt class $A_{1}\left(\mathbb{R}^{n} ; E\right)$ provided there exists a positive constant $C$ such that

$$
\int_{B} w(z) d z \leq C \underset{x \in B}{\operatorname{essinf}} w(x)
$$

holds for every ball $B=B\left(x_{B}, r_{B}\right) \subset \mathbb{R}^{n}$ with $2 r_{B}<d\left(x_{B}, E\right)$. Naturally, $A_{1}\left(\mathbb{R}^{n} ; \emptyset\right)$ stands for the Muckenhoupt class $A_{1}\left(\mathbb{R}^{n}\right)$. Accordingly, $E$ is called the degeneracy set of $w$ when $w \in A_{1}\left(\mathbb{R}^{n} ; E\right)$.

The main result of this paper is the following theorem.

Theorem 1.3. Given $n \geq 2$, let $f: \mathbb{R}^{n} \rightarrow \mathbb{R}^{n}$ be a quasiconformal mapping with $J_{f} \in A_{1}\left(\mathbb{R}^{n} ;\right.$ E) for some closed set $E \subseteq \mathbb{R}^{n}$. If $E$ is a bounded set with $\overline{\operatorname{dim}}_{L} E \in[0, n)$ or $E$ is an unbounded set with $\overline{\operatorname{dim}}_{L G} E \in[0, n)$, then $\mathbf{C}_{f}$ is bounded on $Q_{\alpha}\left(\mathbb{R}^{n}\right)$ for all

$$
0<\alpha< \begin{cases}\min \left\{1, \frac{n-\overline{\operatorname{dim}}_{L} E}{\frac{2}{2}}\right\}, & \text { if } E \text { is bounded; } \\ \min \left\{1, \frac{n-\overline{\operatorname{dim}}_{L G} E}{2}\right\}, & \text { if } E \text { is unbounded. }\end{cases}
$$

In particular, if $E$ is a bounded set with $\overline{\operatorname{dim}}_{L} E \in[0, n-2]$ or $E$ is a unbounded set with $\overline{\operatorname{dim}}_{L G} E \in$ $[0, n-2]$, then $\mathbf{C}_{f}$ is bounded on $Q_{\alpha}\left(\mathbb{R}^{n}\right)$ for all $\alpha \in(0,1)$.

Theorem 1.3 is essentially sharp, see Theorems 1.6 and 1.7 below.

As the first important consequence of Theorem 1.3, we have the following result.

Corollary 1.4. Let $\alpha \in(0,1)$ and $0 \neq \beta \in \mathbb{R}$. If $f(z)=|z|^{\beta-1} z$, then $\mathbf{C}_{f}$ is bounded on $Q_{\alpha}\left(\mathbb{R}^{n}\right)$. In particular, $Q_{\alpha}\left(\mathbb{R}^{n}\right)$ is conformally invariant in the sense that $g \in Q_{\alpha}\left(\mathbb{R}^{n}\right)$ if and only if $x \mapsto g\left(x|x|^{-2}\right)$ is in $Q_{\alpha}\left(\mathbb{R}^{n}\right)$.

Furthermore, for the Tukia-Väisälä quasiconformal extension $f: \mathbb{R}^{n} \rightarrow \mathbb{R}^{n}$ of an arbitrary quasiconformal (quasisymmetric) mapping $g: \mathbb{R}^{n-p} \rightarrow \mathbb{R}^{n-p}$, we obtain the second important consequence of Theorem 1.3.

Corollary 1.5. Given $1 \leq p<n$, suppose $g: \mathbb{R}^{n-p} \rightarrow \mathbb{R}^{n-p}$ is a quasiconformal mapping when $n-p \geq 2$, or a quasisymmetric mapping when $n-p=1$. Let $f: \mathbb{R}^{n} \rightarrow \mathbb{R}^{n}$ be the Tukia-Väisälä's quasiconformal extension of $g$ as in [9]. Then the following hold: 
(i) $J_{f}, J_{f^{-1}} \in A_{1}\left(\mathbb{R}^{n} ; \mathbb{R}^{n-p}\right)$;

(ii) $\mathbf{C}_{f}, \mathbf{C}_{f^{-1}}$ are bounded on $Q_{\alpha}\left(\mathbb{R}^{n}\right)$ for all

$$
0<\alpha<\left\{\begin{array}{lll}
\frac{1}{2} & \text { when } \quad p=1 \\
1 & \text { when } \quad p \geq 2 .
\end{array}\right.
$$

Consequently, $u \in Q_{\alpha}\left(\mathbb{R}^{n}\right)$ if and only if $u \circ f \in Q_{\alpha}\left(\mathbb{R}^{n}\right)$.

The proof of Theorem 1.3 relies on a new characterization of $Q$-spaces established in Section 3 . This technical result allows us to employ our Muckenhoupt assumption and the control on the number of Whitney-type balls guaranteed by our dimension estimate. We expect that our approach will allow one to handle various other function spaces as well.

Our assumption on the control of the fractal size of the degerancy set, whenever which is bounded or unbounded, is necessary in the following sense.

Theorem 1.6. Let $n \geq 2$ and $0<\alpha_{0}<1$. There is a bounded set $E_{\alpha_{0}}$ with $\overline{\operatorname{dim}}_{L} E_{\alpha_{0}}=n-2 \alpha_{0}$ and a quasiconformal (Lipschitz) mapping $f: \mathbb{R}^{n} \rightarrow \mathbb{R}^{n}$ with $J_{f} \in A_{1}\left(\mathbb{R}^{n} ; E_{\alpha_{0}}\right)$ for which $\mathbf{C}_{f}$ is not bounded on $Q_{\alpha}\left(\mathbb{R}^{n}\right)$ for any $\alpha \in\left(\alpha_{0}, 1\right)$.

The main idea in the constructions for Theorem 1.6 is to patch up suitable pieces of radial stretchings in a family of pairwise disjoint balls. In this manner, we also construct an unbounded set $\widetilde{E}_{\alpha_{0}} \subset \mathbb{Z}^{n}$ with $\overline{\operatorname{dim}}_{L G} \widetilde{E}_{\alpha_{0}}=n-2 \alpha$ but $\overline{\operatorname{dim}}_{L} \widetilde{E}_{\alpha_{0}}=0$ and an associated quasiconformal mapping as in Theorem 1.6; see below. This also shows the need for $\overline{\operatorname{dim}}_{L G}$ in Theorem 1.3.

Theorem 1.7. Let $n \geq 2$ and $0<\alpha_{0}<1$. There exists a unbounded set $\widetilde{E}_{\alpha_{0}} \subset \mathbb{Z}^{n}$ with $\overline{\operatorname{dim}}_{L G} \widetilde{E}_{\alpha_{0}}=n-$ $2 \alpha_{0}$ but $\overline{\operatorname{dim}}_{L} \widetilde{E}_{\alpha_{0}}=0$, and a quasiconformal (Lipschitz) mapping $f: \mathbb{R}^{n} \rightarrow \mathbb{R}^{n}$ with $J_{f} \in A_{1}\left(\mathbb{R}^{n} ; \widetilde{E}_{\alpha_{0}}\right)$ for which $\mathbf{C}_{f}$ is not bounded on $Q_{\alpha}\left(\mathbb{R}^{n}\right)$ for any $\alpha \in\left(\alpha_{0}, 1\right)$.

This paper is organized as follows: Section 2 clarifies the relationship between the Minkowski dimension and the local Minkowski dimension $\overline{\operatorname{dim}}_{L}$ or the global Minkowski dimension $\overline{\operatorname{dim}}_{L G}$ and also computes $\overline{\operatorname{dim}}_{L}$ and $\overline{\operatorname{dim}}_{L G}$ for the sets in Theorems 1.6 and 1.7; Section 3 explores a new aspect of $Q_{\alpha}\left(\mathbb{R}^{n}\right)$, which will be used in the proof of Theorem 1.3; in Section 4, we prove Theorem 1.3; Section 5 contains the proofs of Corollaries 1.4 and 1.5; Section 6 is devoted to the proofs of Theorems 1.6 and 1.7 .

Finally, as the converse of the above open question, given a homeomorphism $f: \mathbb{R}^{n} \rightarrow \mathbb{R}^{n}$ for which the composition operator $\mathbf{C}_{f}$ is a bounded on $Q_{\alpha}\left(\mathbb{R}^{n}\right)$ for some $\alpha \in(0,1)$, one would like to know if $f$ is necessarily quasiconformal. The answer is actually in the positive, at least under suitable regularity assumptions on the homeomorphism in question. Since this requires some work, the details will be given in a forthcoming paper.

Notation. In the sequel, we denote by $C$ a positive constant which is independent of the main parameters, but may vary from line to line. The symbol $A \lesssim B$ or $B \gtrsim A$ means that $A \leq C B$. If $A \lesssim B$ and $B \lesssim A$, we then write $A \sim B$. For any locally integrable function $u$ and measurable set $X$, we denote by $f_{X} u$ the average of $u$ on $X$, namely, $f_{X} u \equiv \frac{1}{|X|} \int_{X} u d x$. For a set $\Omega$ and $x \in \mathbb{R}^{n}$, we use $d(x, \Omega)$ to denote $\inf _{z \in \Omega}|x-z|$, the distance from $x$ to $\Omega$. For $\lambda Q$, we mean the cube concentric with $Q$, with sides parallel to the axes, and with length $\ell(\lambda Q)=\lambda \ell(Q)$; similarly, $\lambda B$ denotes the ball concentric with $Q$ with radius $\lambda r_{B}$, where $r_{B}$ is the radius of $B$. 


\section{Local AND GLOBal MinKowsKi Dimensions}

In this section, we clarify the relation between the Minkowski dimension and the above dimensions $\overline{\operatorname{dim}}_{L}$ and $\overline{\operatorname{dim}}_{L G}$. Recall that for a bounded set $E \subset \mathbb{R}^{n}$, its Minkowski dimension $\operatorname{dim}_{M} E$ is defined by

$$
\varlimsup_{\operatorname{dim}}^{M} E=\limsup _{r \rightarrow 0} \frac{\log N_{\text {cov }}(r, E)}{\log (1 / r)},
$$

where $N_{\text {cov }}(r, E)$ is the minimum number of cubes with edge length $r$ required to cover $E$.

\section{Lemma 2.1.}

(i) For every set $E \subset \mathbb{R}^{n}$ and every $R \geq 1$, we have

$$
\overline{\operatorname{dim}}_{L} E=\liminf _{N \rightarrow \infty} \limsup _{r \rightarrow 0} \sup _{\substack{B \subset \mathbb{R}^{n} \\ N r \leq r_{B} \leq R}} \frac{\log N_{\mathrm{cov}}(r, E \cap B)}{\log \left(r_{B} / r\right)} .
$$

(ii) For every set $E \subset \mathbb{R}^{n}$, we always have

$$
0 \leq \sup _{B} \overline{\operatorname{dim}}_{M}(E \cap B) \leq \overline{\operatorname{dim}}_{L} E \leq \overline{\operatorname{dim}}_{L G} E \leq n,
$$

where the supremum is taken over all balls in $\mathbb{R}^{n}$.

(iii) If $E \subset F$, then $\overline{\operatorname{dim}}_{L} E \leq \overline{\operatorname{dim}}_{L} F$ and $\overline{\operatorname{dim}}_{L G} E \leq \overline{\operatorname{dim}}_{L G} F$.

Proof. (i) From the definition, we always have

$$
\overline{\operatorname{dim}}_{L} E \leq \liminf _{N \rightarrow \infty} \limsup _{r \rightarrow 0} \sup _{\substack{B \in \mathbb{R}^{n} \\ N r \leq r_{B} \leq R}} \frac{\log N_{\text {cov }}(r, E \cap B)}{\log \left(r_{B} / r\right)} .
$$

Towards the reverse inequality, notice that every ball $B$ of radius $1 \leq r_{B} \leq R$ can be covered by $c_{n} R^{n}$ balls $B_{i}$ of radii 1 . So

$$
N_{\text {cov }}(r, E \cap B) \leq c_{n} R^{n} \sup \left\{N_{\operatorname{cov}}(r, E \cap \widetilde{B}): \widetilde{B} \subset \mathbb{R}^{n} \text { with } r_{\widetilde{B}}=1\right\}
$$

and hence for all $r<r_{B} / N$ and $r<1$, we have

$$
\frac{\log N_{\mathrm{cov}}(r, E \cap B)}{\log r_{B} / r} \leq \frac{\log c_{n} R^{n}}{\log N}+\sup _{\substack{\widetilde{B} \mathbb{C}^{n} \\ r_{\widetilde{B}}=1}} \frac{\log N_{\mathrm{cov}}(r, E \cap \widetilde{B})}{\log (1 / r)} .
$$

Since the first term on the right-hand side tends to 0 as $N \rightarrow \infty$, by the definition of $\overline{\operatorname{dim}}_{L} E$, we obtain the desired inequality.

(ii) Obviously, $\overline{\operatorname{dim}}_{L G}(E) \leq n$ is obtained from $N_{\text {cov }}(r, E \cap B) \leq\left(2 r_{B} / r\right)^{n}$ for every ball $B$ with radius $r_{B} \geq N r$; indeed,

$$
\overline{\operatorname{dim}}_{L G} E \leq \liminf _{N \rightarrow \infty} \sup _{r>0} \sup _{\substack{B \subset \mathbb{R}^{n} \\ r_{B} \geq N r}} \frac{n \log \left(r_{B} / r\right)+n}{n \log \left(r_{B} / r\right)}=\liminf _{N \rightarrow \infty} \frac{n \log N+n}{n \log N}=n .
$$

The other inequalities follow from the definitions and (i) directly.

(iii) These statements are trivial. 
If $E$ is a set of finitely many points, observing that $N_{\text {cov }}(r, E \cap B) \lesssim 1$ for every ball $B$ with radius $r_{B} \geq N r$, we obtain

which implies that

$$
\varlimsup_{\operatorname{dim}}^{L G} E \leq \liminf _{N \rightarrow \infty} \frac{\log C}{\log N}=0,
$$

$$
\overline{\operatorname{dim}}_{M} E=\overline{\operatorname{dim}}_{L} E=\overline{\operatorname{dim}}_{L G} E=0 .
$$

However, for a countable set $E, \overline{\operatorname{dim}}_{L G}, \overline{\operatorname{dim}}_{L}$ and $\sup _{B} \overline{\operatorname{dim}}_{M}(E \cap B)$ may be very different. Write $(\mathbb{N})^{n}=\mathbb{N} \times \cdots \times \mathbb{N}$ and $\left(2^{\mathbb{N}}\right)^{n}=2^{\mathbb{N}} \times \cdots \times 2^{\mathbb{N}}$ with $2^{\mathbb{N}}=\left\{2^{k}: k \in \mathbb{N}\right\}$. For $\theta \in[0,1]$, set

$$
2^{\mathbb{N}_{\theta}}:=\bigcup_{k \in \mathbb{N} \cup\{0\}} A_{k, \theta}:=\bigcup_{k \in \mathbb{N} \cup\{0\}}\left\{2^{k}, 2^{k}+1, \cdots, 2^{k}+2^{[\theta k]}\right\} .
$$

where $[\theta k]$ is the largest integer less than or equal to $\theta k$. Write $\left(2^{\mathbb{N}_{\theta}}\right)^{n}=2^{\mathbb{N}_{\theta}} \times \cdots \times 2^{\mathbb{N}_{\theta}}$. Observe that

$$
\left\{\begin{array}{l}
2^{\mathbb{N}_{\theta}}=\mathbb{N} \cup\{0\} \text { when } \theta=1 ; \\
2^{\mathbb{N}_{\theta}}=2^{\mathbb{N}} \cup\{1\} \text { when } \theta=0 .
\end{array}\right.
$$

We always have

$$
\overline{\operatorname{dim}}_{M}\left(\left(2^{\mathbb{N}_{\theta}}\right)^{n} \cap B\right)=\overline{\operatorname{dim}}_{L}\left(\left(2^{\mathbb{N}_{\theta}}\right)^{n} \cap B\right)=\overline{\operatorname{dim}}_{L G}\left(\left(2^{\mathbb{N}_{\theta}}\right)^{n} \cap B\right)=0
$$

for all balls $B$ and all $\theta \in[0,1]$ since $\left(2^{\mathbb{N}_{\theta}}\right)^{n} \cap B$ only contains finitely many points.

Lemma 2.2. Let $\theta \in[0,1]$. Then

$$
\overline{\operatorname{dim}}_{L G}\left(2^{\mathbb{N}_{\theta}}\right)^{n}=\theta n ;
$$

in particular, $\overline{\operatorname{dim}}_{L G}\left(2^{\mathbb{N}}\right)^{n}=0$ and $\overline{\operatorname{dim}}_{L G} \mathbb{N}^{n}=n$. But $\overline{\operatorname{dim}}_{L}\left(2^{\mathbb{N} \theta}\right)^{n}=0$.

Proof. We first show that $\overline{\operatorname{dim}}_{L}\left(2^{\mathbb{N}_{\theta}}\right)^{n}=0$. Observe that each $B \subset \mathbb{R}^{n}$ with $r_{B} \leq 1$ contains at most a uniform number of points in $\mathbb{Z}^{n}$. So for each $N \geq 1$ and $r \in\left(0, r_{B} / N\right)$, we can cover $B \cap\left(2^{\mathbb{N} \theta}\right)^{n}$ by a uniform number of balls of radii $r$, that is, $N_{\text {cov }}\left(1,\left(2^{\mathbb{N}_{\theta}}\right)^{n} \cap B\right) \lesssim 1$, which implies that $\operatorname{dim}_{L}\left(2^{\mathbb{N}_{\theta}}\right)^{n} \leq 0$ by definition. So by Lemma $2.1, \overline{\operatorname{dim}}_{L}\left(2^{\mathbb{N}_{\theta}}\right)^{n}=0$.

To show (2.2), we first consider the easy cases $\overline{\operatorname{dim}}_{L G} \mathbb{N}^{n}=n$ and $\overline{\operatorname{dim}}_{L G}\left(2^{\mathbb{N}}\right)^{n}=0$. Indeed, for every ball $B \subset \mathbb{R}^{n}$ with $r_{B}=N$, we have

$$
N_{\text {cov }}\left(1, \mathbb{N}^{n} \cap B\right)=\sharp\left(\mathbb{N}^{n} \cap B\right) \geq(N / \sqrt{n})^{n},
$$

which implies that

$$
\overline{\operatorname{dim}}_{L G} \mathbb{N}^{n} \geq \liminf _{N \rightarrow \infty} \frac{\log (N / \sqrt{n})^{n}}{\log N}=n,
$$

and hence, by Lemma 2.1, $\overline{\operatorname{dim}}_{L G} \mathbb{N}^{n}=n$.

On the other hand, for each $N$ and $r>0$, if $r \leq 1$ and $N r<r_{B}$, we have

$$
N_{\text {cov }}\left(r,\left(2^{\mathbb{N}}\right)^{n} \cap B\right) \leq\left(\log r_{B}\right)^{n} ;
$$

if $2^{k}<r \leq 2^{k+1}$ for some $k \geq 0$, we have

$$
N_{\text {cov }}\left(r,\left(2^{\mathbb{N}}\right)^{n} \cap B\right) \leq \sqrt{n}\left[\log \left(r_{B} / r+2\right)\right]^{n} .
$$

Hence

$$
\overline{\operatorname{dim}}_{L G}\left(2^{\mathbb{N}}\right)^{n} \leq \liminf _{N \rightarrow \infty} \sup _{r \geq 1} \sup _{B, r_{B} \geq N r} \frac{n \log \left[\sqrt{n} \log \left(r_{B} / r+2\right)\right]}{\log \left(r_{B} / r\right)}=0 .
$$


So by Lemma 2.1, we have $\overline{\operatorname{dim}}_{L G}\left(2^{\mathbb{N}}\right)^{n}=0$.

Generally, we let $\theta \in(0,1)$. For every ball $B=B\left(0, \sqrt{n} 2^{m+1}\right)$ with $m \geq 2 / \theta+1$, we have

$$
\left.N_{\text {cov }}\left(1,\left(2^{\mathbb{N} \theta}\right)^{n} \cap B\right)\right) \geq \sharp\left[\left(2^{\mathbb{N}_{\theta}}\right)^{n} \cap B\right] \geq 2^{n \theta m}
$$

an hence,

$$
\overline{\operatorname{dim}}_{L G}\left(2^{\mathbb{N} \theta}\right)^{n} \geq \liminf _{N \rightarrow \infty} \sup _{2^{m+1} \geq N} \frac{n \theta m}{(m+1)}=\liminf _{N \rightarrow \infty} \frac{n \theta(\log N)-n \theta}{\log N}=n \theta .
$$

The proof of $\overline{\operatorname{dim}}_{L G}\left(2^{\mathbb{N}_{\theta}}\right)^{n} \leq \theta n$ is reduced to verifying that for every large $N$, all $r>0$ and all balls $B$ with $r_{B} \geq N r$, we have

$$
\left.N_{\text {cov }}\left(r,\left(2^{\mathbb{N}_{\theta}}\right)^{n} \cap B\right)\right) \lesssim\left(r_{B} / r\right)^{\theta n}
$$

Indeed, this implies that

$$
\begin{aligned}
\varlimsup_{\operatorname{dim}}\left(2^{\mathbb{N} \theta}\right)^{n} & \leq \liminf _{N \rightarrow \infty} \sup _{r>0} \sup _{B, r_{B} \geq N r} \frac{\log \left[\left(r_{B} / r\right)^{\theta n}\right]+\log C}{\log \left(r_{B} / r\right)} \\
& =\liminf _{N \rightarrow \infty} \frac{(\theta n) \log N+\theta n \log C}{\log N} \\
& =\theta n .
\end{aligned}
$$

To prove (2.3), we consider two cases under the assumption $N \geq 2^{5}$.

Case 1: $0<r \leq 1$. If $r_{B}<2$, then $\left(2^{\mathbb{N} \theta}\right)^{n} \cap B$ contains no more than a uniform number of points and hence

$$
\sharp\left(\left(2^{\mathbb{N} \theta}\right)^{n} \cap B\right) \lesssim 1 \lesssim\left(r_{B} / r\right)^{\theta n} .
$$

If $2^{m}<r_{B} \leq 2^{m+1}$ for some $m>1$, then $\left(2^{\mathbb{N} \theta}\right)^{n} \cap B \subset\left[0,2^{m+2}\right]^{n}$. Notice that the interval [0, $\left.2^{m+2}\right]$ contains at most $\sum_{k=1}^{m+1} 2^{\theta k} \sim 2^{\theta m}$ points of $2^{\mathbb{N}_{\theta}}$, and so we have

$$
\sharp\left(\left(2^{\mathbb{N}_{\theta}}\right)^{n} \cap B\right) \lesssim 2^{\theta m n} \lesssim\left(r_{B} / r\right)^{\theta n},
$$

which implies that

$$
N_{\text {cov }}\left(r,\left(2^{\mathbb{N}_{\theta}}\right)^{n} \cap B\right) \leq \sharp\left(\left(2^{\mathbb{N} \theta}\right)^{n} \cap B\right) \lesssim\left(r_{B} / r\right)^{\theta n} .
$$

Case 2: $r>1$. Assume that $2^{\ell}<r<2^{\ell+1}$. Given a ball $B$ with $r_{B} \geq N r$, assume that $2^{m}<r_{B} \leq$ $2^{m+1}$ for some $m \geq 5+\ell$. Then $\left(2^{\mathbb{N} \theta}\right)^{n} \cap B \subset\left[0,2^{m+2}\right]^{n}$. Observe that $\left[0,2^{\ell}\right]$ can be covered by an interval of length $r$. If $\ell \leq k \leq[\ell / \theta]$, then $\left\{2^{k}, 2^{k}+1, \cdots, 2^{k}+2^{[\theta k]}\right\}$ can be covered by an interval of length $r$. If $k>[\ell / \theta]$, then $\left\{2^{k}, 2^{k}+1, \cdots, 2^{k}+2^{[\theta k]}\right\}$ can be covered by $2^{[\theta k]-\ell}+1$ intervals of length $r$. Thus when $m \leq[\ell / \theta]-2,2^{\mathbb{N}_{\theta}} \cap\left[0,2^{m+2}\right]$ can be covered by $m-\ell+2$ intervals of length $r$. If $m>[\ell / \theta]-2$, then $2^{\mathbb{N}_{\theta}} \cap\left[0,2^{m+2}\right]$ can be covered by $2^{\theta m-\ell}$ intervals of length $r$. In both cases, $2^{\mathbb{N}_{\theta}} \cap\left[0,2^{m+2}\right]$ can be covered by $C 2^{\theta(m-\ell)} \leq C\left(r_{B} / r\right)^{\theta}$ intervals of length $r$. Therefore

$$
N_{\text {cov }}\left(r,\left(2^{\mathbb{N}_{\theta}}\right)^{n} \cap\left[0,2^{m+2}\right]^{n}\right) \lesssim\left(r_{B} / r\right)^{\theta n}
$$

which gives (2.2) as desired.

Remark 2.3. Lemma 2.2 indicates that the dimension $\overline{\operatorname{dim}}_{L G}$ not only measures the local selfsimilarity and local Minkowski size but also measures the global selfsimilarity of $E$. 
For a slight modification of the standard Cantor construction, we obtain $E_{a}$ and its self-similar extension $\mathcal{E}_{a}$ so that $\overline{\operatorname{dim}}_{L}$ and $\overline{\operatorname{dim}}_{L G}$ are the same and coincide with $\operatorname{dim}_{M} E_{a}$. Precisely, the sets $E_{a}$ and $\mathcal{E}_{a}$ are defined as follows. Let $a \in(0,1)$. Let $I_{i}, i=1,2$, be the two closed intervals obtained by removing the middle open interval of length $a$ from $I_{0}=[0,1]$ ordered from left to right; when $m \geq 2$, the subintervals $I_{i_{1} \cdots i_{m}}, i_{m}=1,2$, are the two closed intervals obtained by removing the middle open intervals of length $a[(1-a) / 2]^{m-1}$ from $I_{i_{1} \cdots i_{m-1}}$ ordered from left to right. Notice that $\left|I_{i_{1} \cdots i_{m}}\right|=[(1-a) / 2]^{m}$ for $m \geq 1$. For each $m \geq 1$, set

$$
I_{a}^{m}=\bigcup_{i_{1}, \cdots, i_{m} \in\{1,2\}} I_{i_{1} \cdots i_{m}} \& E_{a}^{m}=\left(I_{a}^{m}\right)^{n}=I_{a}^{m} \times \cdots \times I_{a}^{m} .
$$

Notice that $E_{a}^{m}$ consists of $2^{m n}$ disjoint cubes $\left\{Q_{m, j}\right\}_{j=1}^{2 m n}$ with edge length $[(1-a) / 2]^{m}$, and $E_{a}^{m+1} \subset E_{a}^{m}$. Denote by $z_{m, j}$ the center of $Q_{m, j}$ and $z_{0}=\left(\frac{1}{2}, \cdots, \frac{1}{2}\right)$ the center of $Q_{0}=I_{0}^{n}$. Denote by $E_{a}$ the closure of the collection of all these centers, that is,

$$
\left.E_{a}=\overline{\left\{z_{0}, z_{m, j}: m \in \mathbb{N}, j=1, \cdots, 2^{m n}\right.}\right\} .
$$

Set

$$
\mathcal{E}_{a}=\bigcup_{k \geq 0}\left\{\left(\frac{2}{1-a}\right)^{k} x: x \in E_{a}\right\} .
$$

In this case, we consider the larger family $\left\{\widetilde{Q}_{m, j}\right\}_{m \in \mathbb{Z}, j \in \mathbb{N}}$ consisting of all

$$
\left\{\left(\frac{2}{1-a}\right)^{k} x: x \in Q_{m+k, i}\right\}
$$

for all possible $k \geq-m$ and $i=1, \cdots, 2^{(m+k) n}$. Let $\widetilde{z}_{m, j}$ be the center of $\widetilde{Q}_{m, j}$. We also have

$$
\mathcal{E}_{a}=\overline{\left\{\bar{z}_{m, j}: m \in \mathbb{Z}, j \in \mathbb{N}\right\}} \text {. }
$$

Lemma 2.4. For every $a \in(0,1)$,

$$
\overline{\operatorname{dim}}_{M} E_{a}=\overline{\operatorname{dim}}_{L} E_{a}=\overline{\operatorname{dim}}_{L} \mathcal{E}_{a}=\overline{\operatorname{dim}}_{L G} E_{a}=\overline{\operatorname{dim}}_{L G} \mathcal{E}_{a}=\frac{n}{\log [2 /(1-a)]} .
$$

Proof. By Lemma 2.1, it suffices to show that

$$
\overline{\operatorname{dim}}_{M} E_{a} \geq \frac{n}{\log [2 /(1-a)]} \& \overline{\operatorname{dim}}_{L} \mathcal{E}_{a} \leq \frac{n}{\log [2 /(1-a)]} .
$$

To this end, notice that for each $k>m$, we have

$$
\left.2^{(k-m) n}<N_{\operatorname{cov}}([1-a) / 2]^{k}, E_{a} \cap \widetilde{Q}_{m, j}\right) \leq 2^{(k-m) n}+\sum_{\ell=m}^{k-1} 2^{\ell n}<2^{(k+1-m) n},
$$

where recall that $\ell\left(\widetilde{Q}_{m, j}\right)=[(1-a) / 2]^{m}$. For each $r<[(1-a) / 2]^{m+2}$, picking $k_{r}>m$ such that

$$
[(1-a) / 2]^{k_{r}}<r \leq[(1-a) / 2]^{k_{r}-1} \text {, }
$$

we have

$$
\left.\left.N_{\mathrm{cov}}([1-a) / 2]^{k_{r}+1}, \mathcal{E}_{a} \cap \widetilde{Q}_{m, j}\right) \leq N_{\mathrm{cov}}\left(r, \mathcal{E}_{a} \cap \widetilde{Q}_{m, j}\right)<N_{\mathrm{cov}}([1-a) / 2]^{k_{r}}, \mathcal{E}_{a} \cap \widetilde{Q}_{m, j}\right),
$$


and hence, $N_{\text {cov }}\left(r, \mathcal{E}_{a}\right) \sim 2^{\left(k_{r}-m\right) n}$. In particular, $N_{\text {cov }}\left(r, E_{a}\right) \gtrsim 2^{k_{r} n}$, which implies that

$$
\overline{\operatorname{dim}}_{M} E_{a} \geq \limsup _{r \rightarrow 0} \frac{k_{r} n+\log C}{\log (1 / r)}=\limsup _{k_{r} \rightarrow \infty} \frac{k_{r} n+\log C}{k_{r} \log [2 /(1-a)]+\log C_{1}}=\frac{n}{\log [2 /(1-a)]} .
$$

Moreover, for each ball $B$ with $r_{B} \geq[(1-a) / 2]^{3} r$, there exists a $k_{B} \leq k_{\epsilon}-2$ such that

$$
[(1-a) / 2]^{k_{B}}<r_{B} \leq[(1-a) / 2]^{k_{B}-1} .
$$

Hence

$$
\left.N_{\mathrm{cov}}\left(r, \mathcal{E}_{a} \cap B\right) \leq N_{\mathrm{cov}}([1-a) / 2]^{k_{r}}, \mathcal{E}_{a} \cap B\right) \lesssim 2^{\left(k_{r}-k_{B}\right) n} .
$$

Thus

$$
\begin{aligned}
& \sup _{B, r_{B} \geq[(1-a) / 2]^{-N} r} \frac{\log N_{\mathrm{cov}}\left(r, \mathcal{E}_{a} \cap B\right)}{\log \left(r_{B} / r\right)} \\
\leq & \sup _{0 \leq m \leq k_{r}-N} \frac{\log C_{1} 2^{\left(k_{r}-m\right) n}}{\log [(1-a) / 2]^{m-k_{r}}} \\
\leq & \sup _{0 \leq m \leq k_{r}-N} \frac{n\left(k_{r}-m\right)+\log C_{1}}{\left(k_{r}-m\right) \log [2 /(1-a)]} \\
\leq & \frac{n N+\log C_{1}}{N \log [2 /(1-a)]} \\
\rightarrow & n / \log [2 /(1-a)] \text { as } N \rightarrow \infty .
\end{aligned}
$$

Consequently, we get

$$
\overline{\operatorname{dim}}_{L G} \mathcal{E}_{a} \leq \frac{n}{\log [2 /(1-a)]},
$$

as desired.

\section{A characterization of $Q$-Spaces}

In this section, we characterize membership in $Q$-spaces via oscillations. To do so, let us introduce a couple of concepts. Let $u$ be a measurable function. For $\alpha \in(0,1), q \in(0, \infty)$, and each ball $B=B\left(x_{0}, r\right) \subset \mathbb{R}^{n}$, set

$$
\Psi_{\alpha, q}(u, B)=\sum_{k \geq 0} 2^{2 k \alpha} f_{B\left(x_{0}, r\right)} \inf _{c \in \mathbb{R}}\left\{f_{B\left(x, 2^{-k} r\right)}|u(z)-c|^{q} d z\right\}^{2 / q} d x .
$$

Define the space $Q_{\alpha, q}\left(\mathbb{R}^{n}\right)$ as the collection of $u \in L_{\text {loc }}^{q}\left(\mathbb{R}^{n}\right)$ such that

$$
\|u\|_{Q_{\alpha, q}\left(\mathbb{R}^{n}\right)}=\sup _{x_{0} \in \mathbb{R}^{n}, r>0}\left[\Psi_{\alpha, q}\left(u, B\left(x_{0}, r\right)\right)\right]^{1 / 2}<\infty .
$$

Also, for every ball $B \subset \mathbb{R}^{n}$ and each function $u$ on $B$, set

$$
\Phi_{\alpha}(u, B)=|B|^{2 \alpha / n-1} \int_{B} \int_{B} \frac{|u(x)-u(y)|^{2}}{|x-y|^{n+2 \alpha}} d x d y .
$$

Then $\|u\|_{Q_{\alpha}\left(\mathbb{R}^{n}\right)}=\sup _{B}\left[\Phi_{\alpha}(u, B)\right]^{1 / 2}$, where the supremum is taken over all balls $B \subset \mathbb{R}^{n}$. 
Proposition 3.1. Let $\alpha \in(0,1)$ and $q \in(0,2]$. There exists a constant $C$ such that for all measurable functions $u$ and all balls $B=B\left(x_{0}, r\right)$ one has

$$
C^{-1} \Phi_{\alpha}\left(u, B\left(x_{0}, r / 16\right)\right) \leq \Psi_{\alpha, q}\left(u, B\left(x_{0}, r\right)\right) \leq C \Phi_{\alpha}\left(u, B\left(x_{0}, 16 r\right)\right) .
$$

Consequently,

$$
Q_{\alpha}\left(\mathbb{R}^{n}\right)=Q_{\alpha, q}\left(\mathbb{R}^{n}\right) \quad \text { with } \quad\|\cdot\|_{Q_{\alpha}\left(\mathbb{R}^{n}\right)} \sim\|\cdot\|_{Q_{\alpha, q}\left(\mathbb{R}^{n}\right)} .
$$

To verify Proposition 3.1, we need the following estimate from [6].

Lemma 3.2. Let $\sigma \in(0, \infty)$ and $u \in L_{\mathrm{loc}}^{\sigma}\left(\mathbb{R}^{n}\right)$. Then there is a set $E$ with $|E|=0$ such that for each pair of points $x, y \in \mathbb{R}^{n} \backslash E$ with $|x-y| \in\left[2^{-k-1}, 2^{-k}\right)$ one has

$$
\begin{aligned}
& |u(x)-u(y)| \\
& \lesssim \sum_{j \geq k-2}\left\{\inf _{c \in \mathbb{R}}\left[f_{B\left(x, 2^{-j}\right)}|u(w)-c|^{\sigma} d w\right]^{1 / \sigma}+\inf _{c \in \mathbb{R}}\left[f_{B\left(y, 2^{-j}\right)}|u(w)-c|^{\sigma} d w\right]^{1 / \sigma}\right\} .
\end{aligned}
$$

Proof of Proposition 3.1. By Lemma 3.2, we obtain

$$
\begin{aligned}
& \int_{B(x, 2 r)} \frac{|u(x)-u(y)|^{2}}{|x-y|^{n+2 \alpha}} d y \\
& \leq \sum_{j=-1}^{\infty}\left(2^{-j} r\right)^{-(n+2 \alpha)} \int_{B\left(x, 2^{-j} r\right) \backslash B\left(x, 2^{-j-1} r\right)}|u(x)-u(y)|^{2} d y \\
& \quad \sum_{j=-1}^{\infty}\left(2^{-j} r\right)^{-2 \alpha}\left[\sum_{k \geq j-2} \inf _{c \in \mathbb{R}} f_{B\left(x, 2^{-k} r\right)}|u(w)-c|^{q} d w\right]^{2 / q} \\
& \quad+\sum_{j=-1}^{\infty}\left(2^{-j} r\right)^{-2 \alpha} f_{B\left(x, 2^{-j} r\right) \backslash B\left(x, 2^{-j-1} r\right)}\left[\sum_{k \geq j-2} \inf _{c \in \mathbb{R}} f_{B\left(y, 2^{-k}\right)}|u(w)-c|^{q} d w\right]^{2 / q} d y \\
& =J_{1}(x)+J_{2}(x) .
\end{aligned}
$$

Applying Hölder's inequality and changing the order of summation, we obtain

$$
\begin{aligned}
J_{1}(x) & \lesssim \sum_{j=-1}^{\infty}\left(2^{-j} r\right)^{-2 \alpha} 2^{-j \alpha} \sum_{k \geq j-2} 2^{k \alpha} \inf _{c \in \mathbb{R}}\left[f_{B\left(x, 2^{-k} r\right)}|u(w)-c|^{q} d w\right]^{2 / q} \\
& \lesssim \sum_{k \geq-3} 2^{k \alpha} \sum_{j=-1}^{k}\left(2^{-j} r\right)^{-2 \alpha} 2^{-j \alpha} \inf _{c \in \mathbb{R}}\left[f_{B\left(x, 2^{-k} r\right)}|u(w)-c|^{q} d w\right]^{2 / q} \\
& \lesssim \sum_{k \geq-3}\left(2^{-k} r\right)^{-2 \alpha} \inf _{c \in \mathbb{R}}\left[f_{B\left(x, 2^{-k} r\right)}|u(w)-c|^{q} d w\right]^{2 / q} .
\end{aligned}
$$

Thus,

$$
\begin{aligned}
r^{2 \alpha-n} \int_{B\left(x_{0}, r\right)} J_{1}(x) d x & \lesssim \sum_{k \geq-3} 2^{2 k \alpha} f_{B\left(x_{0}, 8 r\right)} \inf _{c \in \mathbb{R}}\left[f_{B\left(x, 2^{-k} r\right)}|u(w)-c|^{q} d w\right]^{2 / q} d x \\
& \lesssim \Psi_{\alpha, q}\left(u, B\left(x_{0}, 8 r\right)\right) .
\end{aligned}
$$


For $J_{2}$, notice that

$$
\int_{B\left(x_{0}, r\right)} J_{2}(x) d x \lesssim \int_{B\left(x_{0}, 4 r\right)} \sum_{j=-1}^{\infty}\left(2^{-j} r\right)^{-2 \alpha}\left[\sum_{k \geq j-2} \inf _{c \in \mathbb{R}} f_{B\left(y, 2^{-k}\right)}|u(w)-c|^{q} d w\right]^{2 / q} d y .
$$

Then, applying an argument similar to the above estimate for $J_{1}$, we have

$$
r^{2 \alpha-n} \int_{B\left(x_{0}, r\right)} J_{2}(x) d x \lesssim \Psi_{\alpha, q}\left(u, B\left(x_{0}, 8 r\right)\right) .
$$

Combining the estimates on $J_{1}$ and $J_{2}$, we obtain

$$
\Phi_{\alpha}\left(u, B\left(x_{0}, r\right)\right) \lesssim \Psi_{\alpha, q}\left(u, B\left(x_{0}, 8 r\right)\right) .
$$

On the other hand, noticing that for all $x \in \mathbb{R}^{n}, r>0$ and $k \geq 0$ one has

$$
2^{-k} r \leq|x-w|-|x-z| \leq|z-w| \leq|x-w|+|x-z| \leq 2^{-k+3} r
$$

whenever

$$
z \in B\left(x, 2^{-k} r\right) \& w \in B\left(x, 2^{-k+2} r\right) \backslash B\left(x, 2^{-k+1} r\right),
$$

we utilize $q \in(0,2]$ and the Hölder inequality to achieve

$$
\begin{aligned}
\inf _{c \in \mathbb{R}} & {\left[f_{B\left(x, 2^{-k} r\right)}|u(w)-c|^{q} d w\right]^{2 / q} } \\
& \lesssim f_{B\left(x, 2^{-k} r\right)}\left|u(z)-u_{\left.B\left(x, 2^{-k} r\right)\right|^{2} d z}\right| u(z)-u_{\left.B\left(x, 2^{-k+2} r\right) \backslash B\left(x, 2^{-k+1} r\right)\right|^{2} d z}|u(z)-u(w)|^{2} d w d z \\
& \lesssim f_{B\left(x, 2^{-k} r\right)} f_{B\left(x, 2^{-k} r\right)} f_{B\left(x, 2^{-k+2} r\right) \backslash B\left(x, 2^{-k+1} r\right)} \frac{|u(z)-u(w)|^{2}}{|z-w|^{n+2 \alpha}} d w d z \\
& \lesssim\left(2^{-k} r\right)^{2 \alpha} f_{B\left(x, 2^{-k} r\right)} \int_{B\left(x, 2^{-k+2} r\right) \backslash B\left(x, 2^{-k+1} r\right)} \frac{|u(z)-u(w)|^{2}}{|z-w|^{n+2 \alpha}} d w d z . \\
& \lesssim\left(2^{-k} r\right)^{2 \alpha} f_{B\left(x, 2^{-k} r\right)} \int_{B\left(z, 2^{-k+3} r\right) \backslash B\left(z, 2^{-k} r\right)}
\end{aligned}
$$

Thus, by changing the order of the integrals with respect to $d z$ and $d x$,

$$
\begin{aligned}
& \Psi_{\alpha, q}\left(u, B\left(x_{0}, r\right)\right) \\
& \quad \lesssim r^{2 \alpha} \sum_{k \geq 0} f_{B\left(x_{0}, r\right)} f_{B\left(x, 2^{-k} r\right)} \int_{B\left(z, 2^{-k+3} r\right) \backslash B\left(z, 2^{-k} r\right)} \frac{|u(z)-u(w)|^{2}}{|z-w|^{n+2 \alpha}} d w d z d x \\
& \quad \lesssim r^{2 \alpha} \sum_{k \geq 0} f_{B\left(x_{0}, 2 r\right)} f_{B\left(z, 2^{-k} r\right)} \int_{B\left(z, 2^{-k+3} r\right) \backslash B\left(z, 2^{-k} r\right)} \frac{|u(z)-u(w)|^{2}}{|z-w|^{n+2 \alpha}} d w d x d z \\
& \quad \lesssim r^{2 \alpha} \sum_{k \geq 0} f_{B\left(x_{0}, 2 r\right)} \int_{B\left(z, 2^{-k+3} r\right) \backslash B\left(z, 2^{-k} r\right)} \frac{|u(z)-u(w)|^{2}}{|z-w|^{n+2 \alpha}} d w d z
\end{aligned}
$$




$$
\begin{aligned}
& \lesssim r^{2 \alpha-n} \int_{B\left(x_{0}, 2 r\right)} \int_{B(z, 8 r)} \frac{|u(z)-u(w)|^{2}}{|z-w|^{n+2 \alpha}} d w d z \\
& \lesssim \Phi_{\alpha}\left(u, B\left(x_{0}, 16 r\right)\right) .
\end{aligned}
$$

This completes the proof of Proposition 3.1.

\section{Proof of Theorem 1.3}

Here we only prove Theorem 1.3 under the assumption $\operatorname{diam} E<\infty$. The case $\operatorname{diam} E=\infty$ is similar. Without loss of generality, we may assume that $\operatorname{diam} E=1$ and $E \subset B(0,1)$. By Proposition 3.1 , it suffices to show that

$$
\Psi_{\alpha, 2}(u \circ f, B) \lesssim\|u\|_{Q_{\alpha}\left(\mathbb{R}^{n}\right)} \quad \text { for each ball } B=B\left(x_{0}, r\right) .
$$

We divide the argument into two cases.

Case 1: $d\left(x_{0}, E\right) \geq 4 r$. Notice that $B(x, 2 r) \cap E=\emptyset$ for all $x \in B\left(x_{0}, r\right)$. By $J_{f} \in A_{1}\left(\mathbb{R}^{n}, E\right)$, and $J_{f}\left(f^{-1}(z)\right) J_{f^{-1}}(z)=1$ for almost all $z \in \mathbb{R}^{n}$, for all $k \geq 0$ and $x \in B\left(x_{0}, r\right)$, we have

$$
\begin{aligned}
\underset{z \in f\left(B\left(x, 2^{-k} r\right)\right)}{\operatorname{esssup}} J_{f^{-1}}(z) & =\underset{z \in f\left(B\left(x, 2^{-k} r\right)\right)}{\operatorname{esssup}}\left[J_{f}\left(f^{-1}(z)\right)\right]^{-1} \\
& =\left[\operatorname{essinf}_{w \in B\left(x, 2^{-k} r\right)} J_{f}(w)\right]^{-1} \\
& \lesssim \frac{\left|B\left(x, 2^{-k} r\right)\right|}{\left|f\left(B\left(x, 2^{-k} r\right)\right)\right|}
\end{aligned}
$$

Thus,

$$
\begin{aligned}
& f_{B\left(x, 2^{-k} r\right)}|u \circ f(z)-c|^{2} d z \\
& \quad=\frac{\left|f\left(B\left(x, 2^{-k} r\right)\right)\right|}{\left|B\left(x, 2^{-k} r\right)\right|} f_{f\left(B\left(x, 2^{-k} r\right)\right)}|u(z)-c|^{2} J_{f^{-1}}(z) d z \\
& \quad \lesssim f_{f\left(B\left(x, 2^{-k} r\right)\right)}|u(z)-c|^{2} d z .
\end{aligned}
$$

Hence we have

$$
\begin{aligned}
& \Psi_{\alpha, 2}\left(u \circ f, B\left(x_{0}, r\right)\right) \\
& \quad \lesssim \sum_{k \geq 0} 2^{2 k \alpha} f_{B\left(x_{0}, r\right)} \inf _{f \in \mathbb{R}} f_{f\left(B\left(x, 2^{-k} r\right)\right)}|u(z)-c|^{2} d z d x \\
& \quad \lesssim \sum_{k \geq 0} \int_{B\left(x_{0}, r\right)} \frac{|B(x, r)|^{2 \alpha / n-1}}{\left|B\left(x, 2^{-k} r\right)\right|^{2 \alpha / n}} \inf _{c \in \mathbb{R}} f_{f\left(B\left(x, 2^{-k} r\right)\right)}|u(z)-c|^{2} d z d x .
\end{aligned}
$$

Observe that $J_{f} \in A_{1}\left(\mathbb{R}^{n}, E\right)$ also implies that

$$
\frac{\left|f\left(B\left(x, 2^{-k} r\right)\right)\right|}{\left|B\left(x, 2^{-k} r\right)\right|}=f_{B\left(x, 2^{-k} r\right)} J_{f}(z) d z \lesssim \operatorname{essinf}_{z \in B\left(x, 2^{-k} r\right)} J_{f}(z) \lesssim J_{f}(x) \quad \text { for almost all } x \in B,
$$


that is,

$$
\left|B\left(x, 2^{-k} r\right)\right|^{-1} \lesssim J_{f}(x)\left|f\left(B\left(x, 2^{-k} r\right)\right)\right|^{-1} .
$$

Therefore, by this and a change of the variables again,

$$
\begin{aligned}
& \Psi_{\alpha, 2}\left(u \circ f, B\left(x_{0}, r\right)\right) \\
& \quad \lesssim \sum_{k \geq 0} \int_{B\left(x_{0}, r\right)} \frac{|B(x, r)|^{2 \alpha / n-1}}{\left|f\left(B\left(x, 2^{-k} r\right)\right)\right|^{2 \alpha / n}} \inf _{c \in \mathbb{R}} f_{f\left(B\left(x, 2^{-k} r\right)\right)}|u(z)-c|^{2} d z\left[J_{f}(x)\right]^{2 \alpha / n} d x \\
& \lesssim \sum_{k \geq 0} \int_{f\left(B\left(x_{0}, r\right)\right)} \frac{\left|B\left(f^{-1}(x), r\right)\right|^{2 \alpha / n-1}}{\left|f\left(B\left(f^{-1}(x), 2^{-k} r\right)\right)\right|^{2 \alpha / n}} \\
& \quad \times \inf _{c \in \mathbb{R}} f_{f\left(B\left(f^{-1}(x), 2^{-k} r\right)\right)}|u(z)-c|^{2} d z J_{f^{-1}}(x)\left[J_{f}\left(f^{-1}(x)\right)\right]^{2 \alpha / n} d x \\
& \lesssim \sum_{k \geq 0} \int_{f\left(B\left(x_{0}, r\right)\right)} \frac{\left|B\left(f^{-1}(x), r\right)\right|^{2 \alpha / n-1}}{\left|f\left(B\left(f^{-1}(x), 2^{-k} r\right)\right)\right|^{2 \alpha / n}} \inf _{c \in \mathbb{R}} \int_{f\left(B\left(f^{-1}(x), 2^{-k} r\right)\right)}|u(z)-c|^{2} d z\left[J_{f^{-1}}(x)\right]^{1-2 \alpha / n} d x .
\end{aligned}
$$

Now, by (4.1) with $k=0$ and $x=x_{0}$, we have

$$
\underset{x \in f\left(B\left(x_{0}, r\right)\right)}{\operatorname{esssup}} J_{f^{-1}}(x) \lesssim \frac{\left|B\left(x_{0}, r\right)\right|}{\left|f\left(B\left(x_{0}, r\right)\right)\right|} \sim \frac{|B(w, r)|}{|f(B(w, r))|} \quad \forall w \in B\left(x_{0}, r\right),
$$

which further yields that

$$
\begin{aligned}
& \Psi_{\alpha, 2}\left(u \circ f, B\left(x_{0}, r\right)\right) \\
& \quad \lesssim \sum_{k \geq 0} f_{f\left(B\left(x_{0}, r\right)\right)}\left(\frac{\left|f\left(B\left(f^{-1}(x), r\right)\right)\right|}{\left|f\left(B\left(f^{-1}(x), 2^{-k} r\right)\right)\right|}\right)^{2 \alpha / n} \inf _{c \in \mathbb{R}} f_{f\left(B\left(f^{-1}(x), 2^{-k} r\right)\right)}|u(z)-c|^{2} d z d x \\
& \quad \lesssim f_{f\left(B\left(x_{0}, r\right)\right)} \sum_{k \geq 0}\left(\frac{L_{f}\left(f^{-1}(x), r\right)}{L_{f}\left(f^{-1}(x), 2^{-k} r\right)}\right)^{2 \alpha} \inf _{c \in \mathbb{R}} f_{f\left(B\left(f^{-1}(x), 2^{-k} r\right)\right)}|u(z)-c|^{2} d z d x,
\end{aligned}
$$

where

$$
L_{f}(z, r)=\sup \{|f(z)-f(w)|:|z-w| \leq r\} \& L_{f}(z, r)^{n} \sim|f(B(z, r))| .
$$

Moreover, by quasisymmetry of $f$, for all $j \in \mathbb{Z}$ and $z \in \mathbb{R}^{n}$, we have

$$
\sharp\left\{k \in \mathbb{Z}: L_{f}\left(z, 2^{-k} r\right) \in\left[2^{-j-1} L_{f}(z, r), 2^{-j} L_{f}(z, r)\right)\right\} \lesssim 1 .
$$

Recalling that

$$
f\left(B\left(x_{0}, r\right)\right) \subset B\left(f\left(x_{0}\right), L_{f}\left(x_{0}, r\right)\right) \& L_{f}\left(f^{-1}(x), r\right) \leq 2^{N_{2}} L_{f}\left(x_{0}, r\right)
$$

holds for some constant $N_{2} \geq 1$ (independent of $x_{0}, r$; see [5]), we arrive at

$$
\begin{aligned}
& \Psi_{\alpha, 2}\left(u \circ f, B\left(x_{0}, r\right)\right) \\
& \quad \lesssim \sum_{j \geq 0} 2^{2 j \alpha} f_{B\left(f\left(x_{0}\right), L_{f}\left(x_{0}, r\right)\right)} f_{B\left(x, 2^{-j} 2^{N_{2}} L_{f}\left(x_{0}, r\right)\right)}|u(z)-c|^{2} d z d x \\
& \quad \lesssim \Psi_{\alpha, 2}\left(u, B\left(f\left(x_{0}\right), 2^{N_{2}} L_{f}\left(x_{0}, r\right)\right)\right),
\end{aligned}
$$


which together with Proposition 3.1 gives

as desired.

$$
\Psi_{\alpha, 2}\left(u \circ f, B\left(x_{0}, r\right)\right) \lesssim\|u\|_{Q_{\alpha}\left(\mathbb{R}^{n}\right)}^{2}
$$

Case 2: $d\left(x_{0}, E\right)<4 r \leq 4$. Recall that each domain $\Omega$ admits a Whitney decomposition. In particular, for $\Omega=\mathbb{R}^{n} \backslash E$, there exists a collection $W_{\Omega}=\left\{S_{j}\right\}_{j \in \mathbb{N}}$ of countably many dyadic (closed) cubes such that

(i) $\Omega=\cup_{j \in \mathbb{N}} S_{j}$ and $\left(S_{k}\right)^{\circ} \cap\left(S_{j}\right)^{\circ}=\emptyset$ for all $j, k \in \mathbb{N}$ with $j \neq k$;

(ii) $2^{7} \sqrt{n} \ell\left(S_{j}\right) \leq \operatorname{dist}\left(S_{j}, \partial \Omega\right) \leq 2^{9} \sqrt{n} \ell\left(S_{j}\right)$;

(iii) $\frac{1}{4} \ell\left(S_{k}\right) \leq \ell\left(S_{j}\right) \leq 4 \ell\left(S_{k}\right)$ whenever $S_{k} \cap S_{j} \neq \emptyset$.

Assume that $2^{-k_{0}-1} \leq 16 r<2^{-k_{0}}$ for $k \in \mathbb{N}$. For each $k \in \mathbb{Z}$, write

$$
\mathscr{S}_{k}(16 B)=\left\{S_{j} \in W_{\Omega}: S_{j} \cap 16 B \neq \emptyset, 2^{-k} \leq \ell\left(S_{j}\right)<2^{-k+1}\right\} \equiv\left\{S_{k, i}\right\}_{i} .
$$

Notice that there exists a integer $N_{0}$ such that if $k \leq k_{0}-N_{0}$, then $\mathscr{S}_{k}(16 B)=\emptyset$. Indeed, since

$$
\operatorname{dist}\left(S_{k, j}, E\right) \leq 16 r+\operatorname{dist}\left(x_{0}, E\right) \leq 20 r,
$$

by (ii) above, we have $2^{-k} \lesssim 2^{-k_{0}}$ which is as desired. Moreover, letting $\epsilon \in\left(0, n-\operatorname{dim}_{L} E-2 \alpha\right)$, we claim that for all $k \geq k_{0}-N$,

$$
\sharp \mathscr{S}_{k}(16 B) \lesssim 2^{\left(k-k_{0}\right)\left(\overline{\operatorname{dim}}_{L} E+\epsilon\right)} \text {. }
$$

To see this, by the definition of $\overline{\operatorname{dim}}_{L} E$ there exists constants $N_{1} \geq 8$ and $k_{1} \in \mathbb{N}$ such that for all $k \geq k_{1}+k_{0}+N_{1}$, we have

which implies that

$$
\frac{\log N_{\operatorname{cov}}\left(2^{-k}, E \cap 32 B\right)}{\log \left(32 r / 2^{-k}\right)} \leq \overline{\operatorname{dim}}_{L} E+\epsilon,
$$

$$
N_{\text {cov }}\left(2^{-k}, E \cap 32 B\right) \lesssim 2^{\left(k-k_{0}\right)\left(\overline{\operatorname{dim}}_{L} E+\epsilon\right)} .
$$

For every $\delta>0$, denote by $\mathscr{N}_{\text {cov }}(\delta, E \cap 32 B)$ the collection of cubes of edge length $\delta$ required to cover $E \cap 32 B$ and

$$
\sharp \mathscr{N}_{\text {cov }}(\delta, E \cap 32 B)=N_{\text {cov }}(\delta, E \cap 32 B) \text {. }
$$

For $k \geq-N_{0}$ and $S_{k, i} \in \mathscr{S}_{k}(16 B)$, we have $2^{11} \sqrt{n} S_{k, i} \cap E \neq \emptyset$ and hence $S_{k, i}$ intersects some cube $Q \in \mathscr{N}_{\text {cov }}\left(2^{-k}, E \cap 32 B\right)$, which implies that $S_{k, i} \subset 2^{13} n Q$. Also notice that for each cube $Q \in$ $\mathscr{N}_{\text {cov }}\left(2^{-k}, 16 B \cap E\right)$, the cube $2^{13} n Q$ can only contain a uniformly bounded number of $S_{k, i} \in \mathscr{S}_{k}(16 B)$. We conclude that for $k \geq-N_{0}$,

$$
\sharp \mathscr{S}_{a}(16 B) \lesssim N_{\text {cov }}\left(2^{-k}, E \cap 32 B\right) .
$$

This together with (4.5) gives that for $k \geq k_{1}+k_{0}+N_{1}$,

$$
\sharp \mathscr{S}_{k}(16 B) \lesssim 2^{\left(k-k_{0}\right)\left(\overline{\operatorname{dim}}_{L} E+\epsilon\right)} \text {. }
$$

On the other hand, if $k_{0}-N_{0} \leq k \leq k_{1}+k_{0}+N_{1}$, then by $2^{k-k_{0}} \leq 2^{k_{1}+N_{1}+N_{0}} \lesssim 1$ we always have

$$
\sharp \mathscr{S}_{k}(16 B) \lesssim 2^{n\left(k-k_{0}\right)} \lesssim 2^{\left(k-k_{0}\right)\left(\overline{\operatorname{dim}}_{L} E+\epsilon\right)} .
$$

This gives the above claim.

By Proposition 3.1, we have

$$
\Psi_{\alpha, 2}\left(u \circ f, B\left(x_{0}, r\right)\right)
$$




$$
\begin{aligned}
& \lesssim \Phi_{\alpha}\left(u \circ f, B\left(x_{0}, 16 r\right)\right) \\
\lesssim & r^{2 \alpha-n} \sum_{k \geq k_{0}-N_{0}} \sum_{i=1}^{\sharp \mathscr{S}_{k}(16 B)} \int_{S_{k, i}} \int_{B\left(x_{0}, 16 r\right)} \frac{|u \circ f(x)-u \circ f(y)|^{2}}{|x-y|^{n+2 \alpha}} d x d y \\
\lesssim & r^{2 \alpha-n} \sum_{k \geq k_{0}-N_{0}} \sum_{i=1}^{\sharp \mathscr{S}_{k}(16 B)} \int_{S_{k, i}} \int_{2 S_{k, i}} \frac{|u \circ f(x)-u \circ f(y)|^{2}}{|x-y|^{n+2 \alpha}} d x d y \\
& +r^{2 \alpha-n} \sum_{k \geq k_{0}-N_{0}} \sum_{i=1}^{\sharp \mathscr{S}_{k}(16 B)} \int_{S_{k, i}} \int_{B\left(x_{0}, 16 r\right) \backslash 2 S_{k, i}} \frac{|u \circ f(x)-u \circ f(y)|^{2}}{|x-y|^{n+2 \alpha}} d x d y \\
= & P_{1}+P_{2} .
\end{aligned}
$$

For each $S_{k, i}$, let $B_{k, i}$ be the ball centered at $x_{k, i}\left(x_{k, i}\right.$ is the center of $\left.S_{k, i}\right)$ and radius $2 \sqrt{n} \ell\left(S_{k, i}\right)$. Then

$$
2 S_{k, i} \subset B_{k, i} \& \operatorname{dist}\left(x_{k, i}, E\right) \geq 4 \cdot 16 \cdot 2 \sqrt{n} \ell\left(S_{k, i}\right) .
$$

So applying the above Case 1 to $16 B_{k, i}$, we have

$$
\Phi_{\alpha}\left(u \circ f, B_{k, i}\right) \lesssim \Psi_{\alpha, 2}\left(u \circ f, 16 B_{k, i}\right) \lesssim\|u\|_{Q_{\alpha}\left(\mathbb{R}^{n}\right)}^{2} .
$$

This, together with $n-2 \alpha-\overline{\operatorname{dim}}_{L} E-\epsilon>0$, gives

$$
\begin{aligned}
P_{1} & \lesssim r^{2 \alpha-n} \sum_{k \geq k_{0}-N_{0}} \sum_{i=1}^{\sharp \mathscr{S}_{a}(16 B)}\left|B_{k, i}\right|^{1-2 \alpha / n} \Phi_{\alpha}\left(u \circ f, B_{k, i}\right) \\
& \lesssim r^{2 \alpha-n} \sum_{k \geq k_{0}-N_{0}} \sum_{i=1}^{\sharp \mathscr{S}_{a}(16 B)} 2^{-(n-2 \alpha) k}\|u\|_{Q_{\alpha}\left(\mathbb{R}^{n}\right)}^{2} \\
& \lesssim \sum_{k \geq k_{0}-N_{0}} 2^{\left(k-k_{0}\right)\left(\operatorname{dim}_{L} E+\epsilon\right)} 2^{(n-2 \alpha)\left(k_{0}-k\right)}\|u\|_{Q_{\alpha}\left(\mathbb{R}^{n}\right)}^{2} \\
& \lesssim\|u\|_{Q_{\alpha}\left(\mathbb{R}^{n}\right)}^{2} .
\end{aligned}
$$

To estimate $P_{2}$, write

$$
\begin{aligned}
& \int_{S_{k, i}} \int_{B\left(x_{0}, 16 r\right) \backslash 2 S_{k, i}} \frac{|u \circ f(x)-u \circ f(y)|^{2}}{|x-y|^{n+2 \alpha}} d x d y \\
& \quad \sum_{\ell=1}^{k-k_{0}+5} 2^{(\ell-k)(-n-2 \alpha)} \int_{S_{k, i}} \int_{2^{\ell+1} S_{k, i} \backslash 2^{\ell} S_{k, i}}|u \circ f(x)-u \circ f(y)|^{2} d x d y \\
& \quad \sum_{\ell=1}^{k-k_{0}+5} 2^{-2 \alpha(\ell-k)} 2^{-k n}\left\{\int_{S_{k, i}}\left|u \circ f(x)-(u \circ f)_{2^{\ell+1} S_{k, i}}\right|^{2} d x\right. \\
& \left.\quad+\int_{2^{\ell+1} S_{k, i}}\left|u \circ f(y)-(u \circ f)_{2^{\ell+1} S_{k, i}}\right|^{2} d y\right\} .
\end{aligned}
$$


Observing that

$$
\begin{aligned}
& \left\{f_{S_{k, i}}\left|u \circ f(x)-(u \circ f)_{2^{\ell+1} S_{k, i}}\right|^{2} d x\right\}^{1 / 2} \\
& \quad \lesssim \sum_{j=1}^{\ell+1}\left\{f_{2^{j} S_{k, i}}\left|u \circ f(x)-(u \circ f)_{2^{j} S_{k, i}}\right|^{2} d x\right\}^{1 / 2} \\
& \quad \lesssim(\ell+1)\|u \circ f\|_{B M O\left(\mathbb{R}^{n}\right)},
\end{aligned}
$$

we obtain

$$
\begin{aligned}
& \int_{S_{k, i}} \int_{B\left(x_{0}, 16 r\right) \backslash 2 S_{k, i}} \frac{|u \circ f(x)-u \circ f(y)|^{2}}{|x-y|^{n+2 \alpha}} d x d y \\
& \quad \lesssim \sum_{\ell=1}^{k-k_{0}+5} 2^{-2 \alpha(\ell-k)} 2^{-k n}(\ell+1)^{2}\|u \circ f\|_{B M O\left(\mathbb{R}^{n}\right)}^{2} \\
& \quad \lesssim 2^{(2 \alpha-n) k}\|u \circ f\|_{B M O\left(\mathbb{R}^{n}\right)}^{2} .
\end{aligned}
$$

Therefore, by $n-2 \alpha-\overline{\operatorname{dim}}_{L} E-\epsilon>0$, one gets

$$
\begin{aligned}
P_{2} & \lesssim r^{2 \alpha-n} \sum_{k \geq k_{0}-N_{0}} \sum_{i=1}^{\sharp \mathscr{S}_{a}(16 B)} 2^{(2 \alpha-n) k)}\|u \circ f\|_{B M O\left(\mathbb{R}^{n}\right)}^{2} \\
& \lesssim \sum_{k \geq k_{0}-N_{0}} 2^{\left(k-k_{0}\right)\left(\overline{\left.\operatorname{dim}_{L} E+\epsilon\right)} 2^{(n-2 \alpha)\left(k_{0}-k\right)}\|u \circ f\|_{B M O\left(\mathbb{R}^{n}\right)}^{2}\right.} \\
& \lesssim\|u \circ f\|_{B M O\left(\mathbb{R}^{n}\right)}^{2} .
\end{aligned}
$$

Recall that it was proved by Reimann [7] that $\|u \circ f\|_{B M O\left(\mathbb{R}^{n}\right)} \lesssim\|u\|_{B M O\left(\mathbb{R}^{n}\right)}$, and also in [3] that $\|u\|_{B M O\left(\mathbb{R}^{n}\right)} \lesssim\|u\|_{Q_{\alpha}\left(\mathbb{R}^{n}\right)}$. Thus $P_{2} \lesssim\|u\|_{Q_{\alpha}\left(\mathbb{R}^{n}\right)}^{2}$.

Combining the estimates for $P_{1}$ and $P_{2}$, we arrive at $\Psi_{\alpha, 2}\left(u \circ f, B\left(x_{0}, r\right)\right) \lesssim\|u\|_{Q_{\alpha}\left(\mathbb{R}^{n}\right)}^{2}$ for all $x_{0}$ and $r$ as desired.

Case 3: $d\left(x_{0}, E\right) \leq 2 r$ and $r>1$. Without loss of generality, we may assume that $x_{0}=0$. Denote by $M$ the minimum number of balls, which are centered in $B(0,1) \backslash B(0,1 / 2)$ and have radius $2^{-9}$, required to cover $B(0,1) \backslash B(0,1 / 2)$. Let $\left\{B_{j}\right\}_{j=1}^{M}$ be a sequence of such balls and write their centers as $\left\{x_{j}\right\}_{j=1}^{M}$. Write

$$
B_{k, j}=B\left(2^{k} x_{j}, 2^{k-9}\right) \text { for } k \geq 2 \text { and } j=1, \cdots, M \text {. }
$$

Notice that

$$
2^{k-9}=2^{k-2} 2^{-7} \leq 2^{-7} d\left(2^{k} x_{j}, E\right) .
$$

Assume that $2^{k_{0}-1} \leq r<2^{k_{0}}$. Then $k_{0} \geq 1$, and $B\left(x_{0}, 16 r\right) \backslash B\left(x_{0}, 2\right)$ can be covered by the family $\left\{B_{k, j}: 2 \leq k \leq k_{0}+4,0 \leq j \leq M\right\}$. Write $B_{1, j}=B(0,2)$. Then we have

$$
\begin{aligned}
& \Psi_{\alpha, 2}\left(u \circ f, B\left(x_{0}, r\right)\right) \\
& \quad \lesssim \Phi_{\alpha}\left(u \circ f, B\left(x_{0}, 16 r\right)\right)
\end{aligned}
$$




$$
\begin{aligned}
\lesssim & \sum_{k=1}^{k_{0}+4} \sum_{j=1}^{M} r^{2 \alpha-n} \int_{B_{k, j}} \int_{16 B} \frac{|u \circ f(x)-u \circ f(y)|^{2}}{|x-y|^{n+2 \alpha}} d x d y \\
\lesssim & \sum_{k=1}^{k_{0}+4} r^{2 \alpha-n} 2^{k(n-2 \alpha)} \Phi_{\alpha}\left(u \circ f, 2 B_{k, j}\right) \\
& +\sum_{k=1}^{k_{0}+4} r^{2 \alpha-n} \int_{B_{k, j}} \int_{16 B \backslash 2 B_{k, j}} \frac{|u \circ f(x)-u \circ f(y)|^{2}}{|x-y|^{n+2 \alpha}} d x d y \\
= & P_{3}+P_{4} .
\end{aligned}
$$

By Proposition 3.1 and the result of Case 1 applied to $32 B_{k, j}$, we have

$$
\Phi_{\alpha}\left(u \circ f, 2 B_{k, j}\right) \lesssim \Psi_{\alpha, 2}\left(u \circ f, 32 B_{k, j}\right) \lesssim\|u\|_{Q_{\alpha}\left(\mathbb{R}^{n}\right)}^{2}
$$

where

$$
32 \cdot 2^{k-9}=32 \cdot 2^{-7} d\left(2^{k} x_{j}, E\right) \leq d\left(2^{k} x_{j}, E\right) / 4
$$

due to (4.6), and hence

$$
P_{3} \lesssim\|u\|_{Q_{\alpha}\left(\mathbb{R}^{n}\right)}^{2} \sum_{k=1}^{k_{0}+4} r^{2 \alpha-n} 2^{k(n-2 \alpha)} \lesssim\|u\|_{Q_{\alpha}\left(\mathbb{R}^{n}\right)}^{2} .
$$

For $P_{4}$, an argument similar to $P_{2}$ in the Case 2 leads to $P_{4} \lesssim\|u\|_{Q_{\alpha}\left(\mathbb{R}^{n}\right)}^{2}$. This finishes the proof of Theorem 1.3.

\section{Proofs of Corollaries 1.4 and 1.5}

Proof of Corollary 1.4. Notice that if $\beta>0$, then $f$ is a quasiconformal mapping from $\mathbb{R}^{n} \rightarrow \mathbb{R}^{n}$, and that

$$
\left\{\begin{array}{l}
J_{f} \in A_{1}\left(\mathbb{R}^{n},\{0\}\right) \text { when } \beta>1 \\
J_{f} \in A_{1}\left(\mathbb{R}^{n}\right) \text { when } 0<\beta<1 .
\end{array}\right.
$$

By Theorem 1.3, if $\beta>0$, then $\mathbf{C}_{f}$ is bounded on $Q_{\alpha}\left(\mathbb{R}^{n}\right)$ for all $\alpha \in(0,1)$. If $\beta<0$, then $f$ is not a quasiconformal mapping from $\mathbb{R}^{n} \rightarrow \mathbb{R}^{n}$; so we can not apply Theorem 1.3 directly. However, observe that $f$ is a quasiconformal mapping from $\mathbb{R}^{n} \backslash\{0\}$ to $\mathbb{R}^{n}$ with $J_{f}(x) \sim|x|^{\beta-1}$ yielding $J_{f} \in A_{1}\left(\mathbb{R}^{n},\{0\}\right)$. Thus, an argument similar to but easier than that for Theorem 1.3 will lead to the boundedness of $\mathbf{C}_{f}$ on $Q_{\alpha}\left(\mathbb{R}^{n}\right)$ for all $\alpha \in(0,1)$.

Indeed, let $u \in Q_{\alpha}\left(\mathbb{R}^{n}\right)$ and $B=B\left(x_{0}, r\right)$ be an arbitrary ball of $\mathbb{R}^{n}$. If $r<\left|x_{0}\right| / 4$, then

$$
J_{f}(x) \sim\left|x_{0}\right|^{\beta-1} \quad \forall \quad x \in B\left(x_{0}, 3 r\right) .
$$

With the help of this and $J_{f} \in A_{1}\left(\mathbb{R}^{n},\{0\}\right)$, similarly to Case 1 in the proof of Theorem 1.3, we obtain (4.2), (4.3) and (4.4). This implies

$$
\Psi_{\alpha, 2}\left(u \circ f, B\left(x_{0}, r\right)\right) \lesssim\|u\|_{Q_{\alpha}\left(\mathbb{R}^{n}\right)}^{2} .
$$

If $r \geq\left|x_{0}\right| / 4$, then by $B\left(x_{0}, r\right) \subset B(0,2 r)$ and Proposition 3.1, we have

$$
\Psi_{\alpha, 2}\left(u \circ f, B\left(x_{0}, r\right)\right) \lesssim \Psi_{\alpha, 2}(u \circ f, B(0,2 r)) \lesssim \Phi_{\alpha}(u \circ f, B(0,32 r)) .
$$


Similarly to Case 3 in the proof of Theorem 1.3, denote by $M$ the minimum number of balls (centered at $B(0,1) \backslash B(0,1 / 2)$ and having radii $\left.2^{-9}\right)$, that are required to cover $B(0,1) \backslash B(0,1 / 2)$. Let $\left\{B_{j}\right\}_{j=1}^{M}$ be a collection of such balls and write their centers as $\left\{x_{j}\right\}_{j=1}^{M}$. Write

$$
B_{k, j}=B\left(2^{-k} 2^{5} r x_{j}, 2^{-k-9} 2^{5} r\right) \text { for } k \geq 0 \text { and } j=1, \cdots, M .
$$

Then $B(0,32 r) \backslash\{0\}$ is covered by the family of balls $\left\{B_{k, j}: k \geq 0,0 \leq j \leq M\right\}$. Therefore, we obtain

$$
\begin{aligned}
& \Psi_{\alpha, 2}\left(u \circ f, B\left(x_{0}, r\right)\right) \\
& \lesssim \sum_{k \geq 0} \sum_{j=1}^{M} r^{2 \alpha-n} \int_{B_{k, j}} \int_{B(0,32 r)} \frac{|u \circ f(x)-u \circ f(y)|^{2}}{|x-y|^{n+2 \alpha}} d x d y \\
& \lesssim \sum_{k \geq 0} r^{2 \alpha-n}\left(2^{-k} r\right)^{n-2 \alpha} \Phi_{\alpha}\left(u \circ f, 2 B_{k, j}\right) \\
& \quad+\sum_{k \geq 0} r^{2 \alpha-n} \int_{B_{k, j}} \int_{B(0,32 r) \backslash 2 B_{k, j}} \frac{|u \circ f(x)-u \circ f(y)|^{2}}{|x-y|^{n+2 \alpha}} d x d y \\
& =P_{5}+P_{6} .
\end{aligned}
$$

Similarly to the estimate on $P_{3}$, we have $P_{5} \lesssim\|u\|_{Q_{\alpha}\left(\mathbb{R}^{n}\right)}^{2}$; and similarly to but easier than for $P_{2}$, we obtain $P_{6} \lesssim\|u\|_{Q_{\alpha}\left(\mathbb{R}^{n}\right)}^{2}$. Putting all together gives

$$
\Psi_{\alpha, 2}\left(u \circ f, B\left(x_{0}, r\right)\right) \lesssim\|u\|_{Q_{\alpha}\left(\mathbb{R}^{n}\right)}^{2},
$$

as desired, and hence finishes the proof of Corollary 1.4.

Proof of Corollary 1.5. For our convenience, let $\mathbb{R}_{+}^{n}=\left\{z=(x, y): x \in \mathbb{R}^{n-1} \& y>0\right\}$. We also write $\mathbb{H}^{n}=\mathbb{R}_{+}^{n} \backslash \mathbb{R}^{n-1}$ and equip it with the hyperbolic distance $d_{\mathbb{H}^{n}}$ - that is -

$$
d_{\mathbb{H}^{n}}\left(w, w^{\prime}\right)=\inf _{\gamma} \int_{\gamma} \frac{|d z|}{y} \quad \forall \quad w, w^{\prime} \in \mathbb{H}^{n},
$$

where the infimum is taken over all rectifiable curves $\gamma$ in $\mathbb{H}^{n}$ joining $w$ and $w^{\prime}$.

Suppose that $g: \mathbb{R}^{n-1} \rightarrow \mathbb{R}^{n-1}$ is a quasiconformal mapping when $n \geq 3$, or a quasisymmetric mapping when $n=2$. According to Tukia-Väisälä [9, Theorem 3.11], $g$ can be extended to such a quasiconformal mapping $f: \mathbb{R}_{+}^{n} \rightarrow \mathbb{R}_{+}^{n}$ that

(i) $\left.f\right|_{\mathbb{R}^{n-1}}=g$;

(ii) $\left.f\right|_{\mathbb{H}^{n}}$ is an $L$-biLipschitz with respect to $d_{\mathbb{H}^{n}}$ for some constant $L \geq 1$, i.e.,

$$
\frac{1}{L} d_{\mathbb{H}^{n}}(z, w) \leq d_{\mathbb{H}^{n}}(f(z), f(w)) \leq L d_{\mathbb{H}^{n}}(z, w) \quad \forall \quad w, w^{\prime} \in \mathbb{H}^{n} .
$$

Obviously, such an $f$ can be further extended to a quasiconformal mapping $\widetilde{f}: \mathbb{R}^{n} \rightarrow \mathbb{R}^{n}$ by reflection, that is,

$$
\widetilde{f}(z)=\left\{\begin{array}{l}
f\left(z_{1}, \cdots, z_{n-1},-z_{n}\right) \text { for } z \in \mathbb{R}^{n} \backslash \mathbb{R}_{+}^{n} \\
f(z) \text { for } z \in \mathbb{R}_{+}^{n}
\end{array}\right.
$$


For sake of simplicity, we write $\widetilde{f}$ as $f$, and generally set

$$
\left\{\begin{array}{l}
n \geq 3 \\
2 \leq p<n \\
\mathbb{H}^{n, p}=\mathbb{R}^{n} \backslash \mathbb{R}^{p}=\left\{z=(x, y): x \in \mathbb{R}^{n-p} \& 0 \neq y \in \mathbb{R}^{p}\right\} .
\end{array}\right.
$$

We equip $\mathbb{H}^{n, p}$ with the distance $d_{\mathbb{H}^{n, p}}$, an analog of the hyperbolic distance, via

$$
d_{\mathbb{H}^{n}, p}\left(w, w^{\prime}\right)=\inf _{\gamma} \int_{\gamma} \frac{|d z|}{|(0, y)|} \quad \forall \quad w, w^{\prime} \in \mathbb{H}^{n, p},
$$

where the infimum is taken over all rectifiable curves $\gamma$ in $\mathbb{H}^{n, p}$ joining $w$ and $w^{\prime}$. Suppose that $g: \mathbb{R}^{n-p} \rightarrow \mathbb{R}^{n-p}$ is a quasiconformal mapping when $n-p \geq 2$, or a quasisymmetric mapping when $n-p=1$. In accordance with Tukia-Väisälä's [9, Section 3.13], $g$ can be extended to a quasiconformal mapping $f: \mathbb{R}^{n} \rightarrow \mathbb{R}^{n}$ such that

(i) $\left.f\right|_{\mathbb{R}^{n-p}}=g$;

(ii) $\left.f\right|_{\mathbb{H}^{n, p}}$ is a $L$-biLipschitz with respect to $d_{\mathbb{H}^{n, p}}$ for some constant $L \geq 1$.

Notice that both $f$ and $f^{-1}$ are biLipschitz with respect to $d_{\mathbb{H}^{n, p}}$. We show that $\mathbf{C}_{f}$ is bounded; the case of $\mathbf{C}_{f^{-1}}$ is analogous. By Theorem 1.3 , it suffices to verify $J_{f} \in A_{1}\left(\mathbb{R}^{n} ; \mathbb{R}^{n-p}\right)$. In what follows, we only consider the case $p=1$; the argument can easily be modified to handle the case $p \geq 2$.

First observe that

$$
J_{f}(z) \sim \frac{\left[d\left(f(z), \mathbb{R}^{n-1}\right)\right]^{n}}{|y|^{n}} \text { a.e. } \quad z=(x, y) \in \mathbb{R}^{n} \backslash \mathbb{R}^{n-1},
$$

where $d\left(f(z), \mathbb{R}^{n-1}\right)$ stands for the Euclidean distance from the point $f(z)$ to $\mathbb{R}^{n-1}$. Indeed, upon taking $r>0$ small enough such that

$$
r<|y| / 2 \quad \& \quad L_{f}(z, r) \leq d\left(f(z), \mathbb{R}^{n-1}\right) / 2,
$$

we get

$$
d\left(w, \mathbb{R}^{n-1}\right) \sim d\left(z, \mathbb{R}^{n-1}\right) \sim|y| \quad \& \quad d\left(f(w), \mathbb{R}^{n-1}\right) / 2 \sim d\left(f(z), \mathbb{R}^{n-1}\right) / 2 \quad \forall w \in B(z, r),
$$

which in turn implies

$$
d_{\mathbb{H}^{n}}(z, w) \sim \frac{|z-w|}{|y|} \quad \& \quad d_{\mathbb{H}^{n}}(f(z), f(w)) \sim \frac{|f(z)-f(w)|}{d\left(f(z), \mathbb{R}^{n-1}\right)} \quad \forall \quad w \in B(z, r) .
$$

Therefore

$$
J_{f}(z) \sim|D f(z)|^{n} \sim \frac{\left[d\left(f(z), \mathbb{R}^{n-1}\right)\right]^{n}}{|y|^{n}} \text { a.e. } \quad z \in \mathbb{R}^{n}
$$

as desired.

Now let $B\left(x_{0}, r\right)$ be an arbitrary ball with radius $r \leq\left|y_{0}\right| / 2$ and $z_{0}=\left(x_{0}, y_{0}\right)$. Obviously, we have

$$
|y| / 2 \leq\left|y_{0}\right| \leq 2|y| \quad \forall \quad z=(x, y) \in B\left(z_{0}, r\right) .
$$

Then, it is enough to prove that

$$
d\left(f\left(z_{0}\right), \mathbb{R}^{n-1}\right) \sim d\left(f(z), \mathbb{R}^{n-1}\right) \quad \text { a.e. } \quad z \in B\left(z_{0}, r\right) .
$$


Assuming this holds for the moment, we have

$$
J_{f}(z) \sim \frac{\left[d\left(f\left(z_{0}\right), \mathbb{R}^{n-1}\right)\right]^{n}}{\left|y_{0}\right|^{n}} \quad \text { a.e. } \quad z \in B\left(z_{0}, r\right)
$$

and further

$$
f_{B\left(x_{0}, r\right)} J_{f}(z) d z \sim \frac{\left[d\left(f\left(z_{0}\right), \mathbb{R}^{n-1}\right)\right]^{n}}{\left|y_{0}\right|^{n}} \sim \operatorname{essinf}_{z \in B\left(x_{0}, r\right)} J_{f}(z),
$$

that is, $J_{f} \in A_{1}\left(\mathbb{R}^{n} ; \mathbb{R}^{n-1}\right)$, as desired.

Towards (5.1), note that $f$ is a quasisymmetric mapping. So, there exists a homeomorphism $\eta$ : $[0, \infty) \rightarrow[0, \infty)$ such that

$$
\frac{|f(z)-f(w)|}{\left|f\left(z_{0}\right)-f(w)\right|} \lesssim \eta\left(\frac{|z-w|}{\left|z_{0}-w\right|}\right) \quad \forall \quad w \in \mathbb{R}^{n}
$$

Observe that

$$
\frac{1}{2}\left|z_{0}-w\right| \leq\left|z_{0}-w\right|-\left|z-z_{0}\right| \leq|z-w| \leq\left|z-z_{0}\right|+\left|z_{0}-w\right| \leq 2\left|z_{0}-w\right| \quad \forall \quad w \in \mathbb{R}^{n-1} .
$$

Thus, by taking such a point $w \in \mathbb{R}^{n-1}$ that

$$
\left|f\left(z_{0}\right)-f(w)\right|=d\left(f\left(z_{0}\right), \mathbb{R}^{n-1}\right),
$$

we have

$$
d\left(f(z), \mathbb{R}^{n-1}\right) \leq|f(z)-f(w)| \leq \eta(2)\left|f\left(z_{0}\right)-f(w)\right| \lesssim d\left(f\left(z_{0}\right), \mathbb{R}^{n-1}\right) .
$$

Upon changing the roles of $z$ and $z_{0}$, we also have

$$
d\left(f\left(z_{0}\right), \mathbb{R}^{n-1}\right) \lesssim d\left(f(z), \mathbb{R}^{n-1}\right) .
$$

Hence (5.1) holds. This completes the proof of Corollary 1.5.

\section{Proofs of Theorems 1.6 ANd 1.7}

Proof of Theorem 1.6. Fix $\alpha_{0} \in(0,1)$. Let $a=1-2^{-2 \alpha_{0} /\left(n-2 \alpha_{0}\right)} \in(0,1)$, and let the sets $E_{a}$ be as (2.4) in Section 2. Then we have $n-2 \alpha_{0}=n / \log [2 /(1-a)]$ and by Lemma 2.4, $\overline{\operatorname{dim}}_{L} E_{a}=n-2 \alpha_{0}$. The set $E_{a}$ is exactly what we want in the statement of Theorem 1.6.

Now we are going to construct a quasiconformal (Lipschitz) mapping $f: \mathbb{R}^{n} \rightarrow \mathbb{R}^{n}$ such that $J_{f} \in A_{1}\left(\mathbb{R}^{n}, E_{a}\right)$ and hence $J_{f} \in A_{1}\left(\mathbb{R}^{n}, \mathcal{E}_{a}\right)$ but $\mathbf{C}_{f}$ is unbounded on $Q_{\alpha}\left(\mathbb{R}^{n}\right)$ for any $\alpha \in\left(\alpha_{0}, 1\right)$.

Recall that $\left\{z_{m, j}\right\}$ are the centers of $\left\{Q_{m, j}\right\}$ and $\left\{Q_{m, j}\right\}$ are the pre-cubes appearing in the Cantor construction $E_{a}$, see Section 2. Let $\beta \in(0, \infty)$ and define the map $f$ by setting

$$
f(x)=\left(\frac{1}{2} a[(1-a) / 2]^{m}\right)^{-\beta}\left|x-z_{m, j}\right|^{\beta}\left(x-z_{m, j}\right)+z_{m, j}
$$

if

$$
\left|x-z_{m, j}\right|<\frac{1}{2} a[(1-a) / 2]^{m} \text { for some } m \in \mathbb{N} \text { and } j=1, \cdots, 2^{m n},
$$

and $f(x)=x$ otherwise. Indeed, we only perturb the identity mapping on all balls

$$
B\left(z_{m, j}, \frac{1}{2} a[(1-a) / 2]^{m}\right) \subset Q_{m, j}
$$


by making "radial" stretchings with respect to their centers, where $\left|Q_{m, j}\right|=a[(1-a) / 2]^{m n}$. Notice that

$$
J_{f}(x) \sim|D f(x)|^{n} \sim\left(\frac{1}{2} a[(1-a) / 2]^{m}\right)^{-n \beta}\left|x-z_{m, j}\right|^{n \beta} \lesssim 1 \text { when }\left|x-z_{m, j}\right|<\frac{1}{2} a[(1-a) / 2]^{m},
$$

and

$$
J_{f}(x)=|D f(x)|^{n}=1 \text { otherwise. }
$$

Thus $f$ is a quasiconformal mapping. Moreover, it is easy to check that

$$
J_{f} \in A_{1}\left(\mathbb{R}^{n} ; E_{a}\right) \& J_{f} \notin A_{1}\left(\mathbb{R}^{n}\right) .
$$

Set

$$
\beta_{0}=1+\frac{n-2 \alpha}{n} \log \left(\frac{1-a}{2}\right)
$$

Then

Set also

$$
\beta_{0}>0 \text { since } n-2 \alpha<n-2 \alpha_{0}=\frac{n}{\log [2 /(1-a)]} \text {. }
$$

$$
\left\{\begin{array}{l}
\ell=m n \beta /(n-2 \alpha) \text { if } 0<\beta \leq \beta_{0} \\
\ell=m n \beta_{0} /(n-2 \alpha) \text { if } \beta>\beta_{0} .
\end{array}\right.
$$

With each $z_{m, j} \in E$, we associate a ball $B_{m, j}$ such that

$$
B_{m, j} \subset \frac{17}{64} 2^{-\ell} a Q_{m, j} \& r_{m, j}=\frac{1}{64} 2^{-\ell} a[(1-a) / 2]^{m}
$$

and so that the center $x_{m, j}$ of $B_{m, j}$ satisfies

$$
\left|x_{m, j}-z_{m, j}\right|=\frac{1}{4} 2^{-\ell} a[(1-a) / 2]^{m} .
$$

For each $m$, set

$$
u_{m}=\sum_{j=1}^{2^{m n}} u_{m, j}
$$

where

$$
u_{m, j}(x)=\chi_{B_{m, j}} d\left(x, \partial B_{m, j}\right) \text { for all possible } j .
$$

Obviously, $u_{m, j}$ is a Lipschitz function.

We make two claims:

$$
\left\|u_{m}\right\|_{Q_{\alpha}\left(\mathbb{R}^{n}\right)}^{2} \lesssim 2^{m n} 2^{-\ell(n+2-2 \alpha)}[(1-a) / 2]^{m(n+2-2 \alpha)}
$$

and

$$
\left\|u_{m} \circ f\right\|_{Q_{\alpha}\left(\mathbb{R}^{n}\right)}^{2} \gtrsim 2^{m n} 2^{-\ell(n-2 \alpha) /(\beta+1)} 2^{-2 \ell}[(1-a) / 2]^{m(n-2 \alpha+2)} .
$$

Assuming that both (6.1) and (6.2) hold for the moment, we arrive at

$$
\frac{\left\|u_{m} \circ f\right\|_{Q_{\alpha}\left(\mathbb{R}^{n}\right)}^{2}}{\left\|u_{m}\right\|_{Q_{\alpha}\left(\mathbb{R}^{n}\right)}^{2}} \gtrsim \frac{2^{m n} 2^{-\ell(n-2 \alpha) /(\beta+1)} 2^{-2 \ell}[(1-a) / 2]^{m(n-2 \alpha+2)}}{2^{m n} 2^{-\ell(n+2-2 \alpha)}[(1-a) / 2]^{m(n+2-2 \alpha)}} \gtrsim 2^{\ell(n-2 \alpha) \beta /(\beta+1)},
$$

which tends to $\infty$ as $m \rightarrow \infty$ since $\beta>0$ and $\ell \sim m$. This gives Theorem 1.6 under (6.1)-(6.2).

Finally, we verify (6.1)-(6.2). 
Proof of (6.1). Let $B=B\left(x_{B}, r_{B}\right)$ be an arbitrary ball.

If $r_{B} \leq r_{m, j}$, since

$$
\left|u_{m}(x)-u_{m}(y)\right| \leq|x-y| \forall x, y \in \mathbb{R}^{n}
$$

one has

$$
\begin{aligned}
\Phi_{\alpha}\left(u_{m}, 2 B\right) & \lesssim r_{B}^{2 \alpha-n} \int_{2 B} \int_{2 B} \frac{1}{|x-y|^{n-2(1-\alpha)}} d x d y \\
& \lesssim r_{B}^{2 \alpha-n} \int_{2 B} \int_{B\left(y, 2 r_{B}\right)} \frac{1}{|x-y|^{n-2(1-\alpha)}} d x d y \\
& \lesssim r_{B}^{2} \lesssim r_{m, j}^{2} .
\end{aligned}
$$

In particular, $\Phi_{\alpha}\left(u_{m}, 2 B_{m, j}\right) \lesssim r_{m, j}^{2}$

If $r_{B}>r_{m, j}$, one writes

$$
\begin{aligned}
\Phi_{\alpha}\left(u_{m}, 2 B\right) \leq & 2|B|^{2 \alpha / n-1} \sum_{B_{m, j} \cap 2 B \neq \emptyset} \int_{B_{m, j}} \int_{2 B} \frac{\left|u_{m}(x)-u_{m}(y)\right|^{2}}{|x-y|^{n+2 \alpha}} d x d y \\
\leq & |B|^{2 \alpha / n-1} \sum_{B_{m, j} \cap 2 B \neq \emptyset}\left|B_{m, j}\right|^{1-2 \alpha / n} \Phi_{\alpha}\left(u_{m}, 2 B_{m, j}\right) \\
& +|B|^{2 \alpha / n-1} \sum_{B_{m, j} \cap 2 B \neq \emptyset} \int_{B_{m, j}} \int_{2 B \backslash 2 B_{m, j}} \frac{\left|u_{m}(x)-u_{m}(y)\right|^{2}}{|x-y|^{n+2 \alpha}} d x d y .
\end{aligned}
$$

Notice that

$$
\begin{aligned}
\int_{B_{m, j}} \int_{2 B \backslash 2 B_{m, j}} \frac{\left|u_{m}(x)-u_{m}(y)\right|^{2}}{|x-y|^{n+2 \alpha}} d x d y & \lesssim r_{m, j}^{2}\left|B_{m, j}\right| \int_{2 B \backslash 2 B_{m, j}} \frac{1}{\left|y-z_{m, j}\right|^{n+2 \alpha}} d y \\
& \lesssim r_{m, j}^{2-2 \alpha}\left|B_{m, j}\right| .
\end{aligned}
$$

So, by (6.3) one has

$$
\Phi_{\alpha}\left(u_{m}, 2 B\right) \lesssim|B|^{2 \alpha / n-1} \sum_{B_{m, j} \cap 2 B \neq \emptyset} r_{m, j}^{2-2 \alpha+n} .
$$

Below we consider three subcases.

First, if $r_{m, j}<r_{B} \leq \frac{1}{64} a[(1-a) / 2]^{m}$, there are a uniformly bounded number of balls $B_{m, j}$ such that $B_{m, j} \cap 2 B \neq \emptyset$ and hence

$$
\Phi_{\alpha}\left(u_{m}, 2 B\right) \lesssim|B|^{2 \alpha / n-1}\left\{2^{-\ell}[(1-a) / 2]^{m}\right\}^{2-2 \alpha+n} \lesssim 2^{-2 \ell}[(1-a) / 2]^{2 m} .
$$

Second, if $\frac{1}{64} a[(1-a) / 2]^{m-k}<r_{B} \leq \frac{1}{64} a[(1-a) / 2]^{m-k-1}$ for some $1 \leq k \leq m$, there are at most $2^{k n}$, up to a constant multiplier, many $B_{m, j}$ such that $B \cap B_{m, j} \neq \emptyset$, and hence

$$
\Phi_{\alpha}\left(u_{m}, 2 B\right) \lesssim[(1-a) / 2]^{(m-k)(2 \alpha-n)} 2^{k n} 2^{-\ell(n+2-2 \alpha)}[(1-a) / 2]^{m(n+2-2 \alpha)} .
$$

Since $2^{n}[(1-a) / 2]^{(n-2 \alpha)}>1$ due to $n-(n-2 \alpha) \log [2 /(1-a)]>0$, we obtain

$$
\Phi_{\alpha}\left(u_{m}, 2 B\right) \lesssim 2^{m n} 2^{-\ell(n+2-2 \alpha)}[(1-a) / 2]^{m(n+2-2 \alpha)} .
$$


Third, if $r_{B}>\frac{1}{64} a[(1-a) / 2]$, there are at most $2^{m n}$, up to a constant multiplier, many $B_{m, j}$ such that $B \cap B_{m, j} \neq \emptyset$, and hence

$$
\Phi_{\alpha}\left(u_{m}, 2 B\right) \lesssim 2^{m n} 2^{-\ell(n+2-2 \alpha)}[(1-a) / 2]^{m(n+2-2 \alpha)} .
$$

To sum up, one obtains

$$
\left\|u_{m}\right\|_{Q_{\alpha}\left(\mathbb{R}^{n}\right)} \lesssim \max \left\{2^{-2 \ell}[(1-a) / 2]^{2 m}, 2^{m n} 2^{-\ell(n+2-2 \alpha)}[(1-a) / 2]^{m(n+2-2 \alpha)}\right\} .
$$

So (6.1) will follow from this if one can show

$$
2^{-2 \ell}[(1-a) / 2]^{2 m} \leq 2^{m n} 2^{-\ell(n+2-2 \alpha)}[(1-a) / 2]^{m(n+2-2 \alpha)} .
$$

Obviously, this is equivalent to

$$
2^{\ell(n-2 \alpha)} \leq 2^{m n}[(1-a) / 2]^{m(n-2 \alpha)},
$$

and hence to

$$
\ell(n-2 \alpha) \leq m n+m(n-2 \alpha) \log [(1-a) / 2] .
$$

But this last estimate follows from our choice of $\ell$, namely,

$$
\ell=\frac{m n}{n-2 \alpha} \min \left\{\beta, \beta_{0}\right\} \leq \frac{m n}{n-2 \alpha} \beta_{0}=\frac{m n}{n-2 \alpha}+m \log \left(\frac{1-a}{2}\right) .
$$

Thus (6.1) holds.

Proof of (6.2). Indeed, we have

$$
\begin{aligned}
\left\|u_{m} \circ f\right\|_{Q_{\alpha}\left(\mathbb{R}^{n}\right)}^{2} & \geq \Phi_{\alpha}\left(u_{m} \circ f, f^{-1}(B(0,2))\right) \\
& \gtrsim \sum_{j=1}^{2^{m n}} \int_{f^{-1}\left(B_{m, j}\right)} \int_{f^{-1}\left(B_{m, j}\right)} \frac{\left|u_{m, j} \circ f(x)-u_{m, j} \circ f(y)\right|^{2}}{|x-y|^{n+2 \alpha}} d x d y \\
& \gtrsim \sum_{j=1}^{2^{m n}}\left|f^{-1}\left(B_{m, j}\right)\right|^{1-2 \alpha / n} \Phi_{\alpha}\left(u_{m, j} \circ f, f^{-1}\left(B_{m, j}\right)\right) .
\end{aligned}
$$

It suffices to estimate

$$
\left|f^{-1}\left(B_{m, j}\right)\right| \& \Phi_{\alpha}\left(u_{m, j} \circ f, f^{-1}\left(B_{m, j}\right)\right)
$$

from below. We first notice that if $\left|x-z_{m, j}\right|<\frac{1}{2} a[(1-a) / 2]^{m}$, then

$$
f^{-1}(x)=\left(\frac{1}{2} a[(1-a) / 2]^{m}\right)^{\beta /(\beta+1)}\left|x-z_{m, j}\right|^{-\beta /(\beta+1)}\left(x-z_{m, j}\right)+z_{m, j}
$$

and hence

$$
J_{f^{-1}}(x) \sim\left(\frac{1}{2} a[(1-a) / 2]^{m}\right)^{n \beta /(\beta+1)}\left|x-z_{m, j}\right|^{-n \beta /(\beta+1)} .
$$

For every $y \in B_{m, j}$, we have that if $\left|x-z_{m, j}\right|=2 r_{m, j}$, then

$$
r_{m, j} \leq\left|z_{m, j}-y_{m, j}\right|-\left|y-y_{m, j}\right| \leq\left|y-z_{m, j}\right| \leq\left|z_{m, j}-y_{m, j}\right|+\left|y-y_{m, j}\right| \leq 3 r_{m, j}
$$

and hence

$$
J_{f^{-1}}(y) \sim\left(\frac{1}{2} a[(1-a) / 2]^{m}\right)^{n \beta /(\beta+1)} r_{m, j}^{-n \beta /(\beta+1)} \sim 2^{\ln \beta /(\beta+1)}
$$


Therefore,

and

$$
\left|f^{-1}\left(B_{m, j}\right)\right| \sim 2^{-\ell n /(\beta+1)}[(1-a) / 2]^{m n}
$$

$$
f_{B_{m, j}} J_{f}(y) d y \sim 2^{\ell n \beta /(\beta+1)} \lesssim \operatorname{essinf}_{y \in B_{m, j}} J_{f}(y) .
$$

Moreover, by $J_{f^{-1}} \in A_{1}\left(\mathbb{R}^{n}\right)$ and similarly to (4.4), we have

$$
\begin{aligned}
\Phi_{\alpha}\left(u_{m, j} \circ f, f^{-1}\left(B_{m, j}\right)\right) & \gtrsim \Psi_{\alpha, 2}\left(u_{m, j} \circ f, f^{-1}\left(2^{-4} B_{m, j}\right)\right) \\
& \gtrsim \Psi_{\alpha, 2}\left(u_{m, j}, 2^{-4-N_{2}} B_{m, j}\right) \\
& \gtrsim \Phi_{\alpha}\left(u_{m, j}, 2^{-8-N_{2}} B_{m, j}\right) .
\end{aligned}
$$

Notice that for all

$$
x \in 2^{-12-N_{2}} B_{m, j} \& y \in 2^{-8-N_{2}} B_{m, j} \backslash 2^{-9-N_{2}} B_{m, j},
$$

we have

$$
|x-y| \sim r_{m, j} \&\left|u_{m, j}(x)-u_{m, j}(y)\right| \geq 2^{-9-N_{2}} r_{m, j}-2^{-12-N_{2}} r_{m, j} \geq 2^{-10-N_{2}} r_{m, j}
$$

Hence,

$$
\begin{aligned}
\Phi_{\alpha}\left(u_{m, j}, 2^{-8} B_{m, j}\right) & \gtrsim r_{m, j}^{2 \alpha-n} f_{2^{-12-N_{2}} B_{m, j}} \int_{2^{-8-N_{2} B_{m, j}} 2^{-9-N_{2} B_{m, j}}} \frac{\left|u_{m, j}(x)-u_{m, j}(y)\right|^{2}}{|x-y|^{n+2 \alpha}} d x d y \\
& \gtrsim r_{m, j}^{2 \alpha-n} r_{m, j}^{2 n} r_{m, j}^{-n-2 \alpha+2} \\
& \gtrsim r_{m, j}^{2} .
\end{aligned}
$$

Therefore

$$
\Phi_{\alpha}\left(u_{m, j} \circ f, f^{-1}\left(B_{m, j}\right)\right) \gtrsim r_{m, j}^{2} .
$$

This together with (6.3) implies that

$$
\begin{aligned}
\left\|u_{m} \circ f\right\|_{Q_{\alpha}\left(\mathbb{R}^{n}\right)}^{2} & \gtrsim 2^{m n} 2^{-\ell(n-2 \alpha) /(\beta+1)}[(1-a) / 2]^{m(n-2 \alpha)} 2^{-2 \ell}[(1-a) / 2]^{2 m} \\
& \sim 2^{m n} 2^{-\ell(n-2 \alpha) /(\beta+1)} 2^{-2 \ell}[(1-a) / 2]^{m(n-2 \alpha+2)}
\end{aligned}
$$

as desired.

Proof of Theorem 1.7. Fix $\alpha_{0} \in(0,1)$. Let $\theta=\left(n-2 \alpha_{0}\right) / n \in(0,1)$ and $\widetilde{E}_{\alpha_{0}}=\left(2^{\mathbb{N}_{\theta}}\right)^{n}$ be as (2.1) in Section 2. By Lemma 2.2, $\overline{\operatorname{dim}}_{L G}\left(2^{\mathbb{N}_{\theta}}\right)^{n}=n-2 \alpha_{0}$ but $\operatorname{dim}_{L}\left(2^{\mathbb{N}_{\theta}}\right)^{n}=0$.

Now we need to construct a quasiconformal (Lipschitz) mapping $f: \mathbb{R}^{n} \rightarrow \mathbb{R}^{n}$ such that $J_{f} \in$ $A_{1}\left(\mathbb{R}^{n},\left(2^{\mathbb{N}_{\theta}}\right)^{n}\right)$ but $\mathbf{C}_{f}$ is unbounded on $Q_{\alpha}\left(\mathbb{R}^{n}\right)$ for each $\alpha \in\left(\alpha_{0}, 1\right)$. The idea is similar to the construction of Theorem 1.6. We divide the argument into two cases.

Case 1: $\alpha_{0}=1$. Let $\beta>0$ and define

$$
\left\{\begin{array}{l}
f(x)=|x-\vec{k}|^{\beta}(x-\vec{k})+\vec{k} \text { if } x \in B(\vec{k}, 1) \text { with } \vec{k} \in(3 \mathbb{N})^{n} \\
f(x)=x \text { if } x \notin \cup_{\vec{k} \in \mathbb{N}^{n}} B(\vec{k}, 1) .
\end{array}\right.
$$

Then $f$ is a quasiconformal mapping and

$$
\left\{\begin{array}{l}
J_{f}(x) \sim|x-\vec{k}|^{n \beta} \text { if } x \in B(\vec{k}, 1) \text { for some } \vec{k} \in(3 \mathbb{N})^{n} \\
J_{f}(x)=1 \text { otherwise. }
\end{array}\right.
$$


Now we show that $\mathbf{C}_{f}$ is unbounded on $Q_{\alpha}\left(\mathbb{R}^{n}\right)$ for each $\alpha \in(0,1)$. Indeed, for each $\vec{k} \in(3 \mathbb{N})^{n}$, we take a ball $B_{\vec{k}}$ such that

Set

$$
\left|x_{B_{\vec{k}}}-\vec{k}\right|=2^{-m} \& r_{B_{\vec{k}}}=2^{-m-5} \text {. }
$$

For each $m$, set

$$
u_{\vec{k}}(x)=\chi_{B_{\vec{k}}} d\left(x, \partial B_{\vec{k}}\right) .
$$

$$
u_{m}=\sum_{|\vec{k}| \leq 2^{\ell}} u_{\vec{k}} \text { with } \ell=m(n-2 \alpha) / 2 \alpha .
$$

Observe that if $x \in B_{\vec{k}}$, then

$$
f^{-1}(x)=|x-\vec{k}|^{-\beta /(\beta+1)}\left(x-x_{\vec{k}}\right)+x_{\vec{k}}
$$

and hence

$$
J_{f^{-1}}(x) \sim|x-\vec{k}|^{-n \beta /(\beta+1)} \sim 2^{m \beta /(\beta+1)} .
$$

Thus, one gets $\left|f^{-1}\left(B_{\vec{k}}\right)\right| \sim 2^{-[1-\beta /(\beta+1)] m n}$.

By an argument similar to (6.5) for $\Phi_{\alpha}\left(u_{m, j} \circ f, f^{-1}\left(B_{m, j}\right)\right)$, we have

$$
\Phi_{\alpha}\left(u_{\vec{k}} \circ f, f^{-1}\left(B_{\vec{k}}\right)\right) \gtrsim 2^{-2 m} \text {. }
$$

This leads to

$$
\begin{aligned}
\left\|u_{m} \circ f\right\|_{Q_{\alpha}\left(\mathbb{R}^{n}\right)}^{2} & \geq \Phi_{\alpha}\left(u_{m} \circ f, f^{-1}\left(B\left(0,2^{\ell+1}\right)\right)\right) \\
& \gtrsim 2^{\ell(2 \alpha-n)} \sum_{|\vec{k}| \leq 2^{\ell}}\left|f^{-1}\left(B_{\vec{k}}\right)\right|^{1-2 \alpha / n} \Phi_{\alpha}\left(u_{\vec{k}} \circ f, f^{-1}\left(B_{\vec{k}}\right)\right) \\
& \gtrsim 2^{\ell(2 \alpha-n)} \sum_{|\vec{k}| \leq 2^{\ell}} 2^{-2 m} 2^{-[1-\beta /(\beta+1)] m(n-2 \alpha)} \\
& \gtrsim 2^{2 \alpha \ell} 2^{-2 m} 2^{-[1-\beta /(\beta+1)] m(n-2 \alpha)} \\
& \gtrsim 2^{-2 m} 2^{m(n-2 \alpha) \beta /(\beta+1)},
\end{aligned}
$$

where $\ell=m(n-2 \alpha) / 2 \alpha$.

On the other hand, we claim that $\left\|u_{m}\right\|_{Q_{\alpha}\left(\mathbb{R}^{n}\right)}^{2} \lesssim 2^{-2 m}$. The proof of this estimate is similar to that of (6.1). Five situations are required to handle.

If $r_{B} \leq 2^{-m-5}$, by an argument similar to (6.3), we have $\Phi_{\alpha}\left(u_{m}, 2 B\right) \lesssim 2^{-2 m}$.

If $r_{B}>2^{-m-5}$, similarly to (6.4), we also have

$$
\Phi_{\alpha}\left(u_{m}, 2 B\right) \lesssim|B|^{2 \alpha / n-1} \sum_{B_{\vec{k}} \cap 2 B \neq \emptyset} 2^{-m(2-2 \alpha+n)} .
$$

If $2^{-m-5}<r_{B} \leq 1$, there is at most one $B_{\vec{k}}$ such that $B \cap B_{\vec{k}} \neq \emptyset$ and hence $\Phi_{\alpha}\left(u_{m}, 2 B\right) \lesssim 2^{-2 m}$.

If $1 \leq r_{B} \leq 2^{\ell}$, then there are at most $2^{n+2} r_{B}^{n}$ many $B_{\vec{k}}$ such that $B \cap B_{\vec{k}} \neq \emptyset$, and hence

$$
\Phi_{\alpha}\left(u_{m}, 2 B\right) \lesssim r_{B}^{n} r_{B}^{2 \alpha-n} 2^{-m(2-2 \alpha+n)} \lesssim 2^{2 \alpha \ell} 2^{-m(2-2 \alpha+n)} \lesssim 2^{-2 m},
$$

where $\ell=m(n-2 \alpha) / 2 \alpha$.

If $r_{B}>2^{\ell}$, then there are at most $2^{n+2} 2^{\ell n}$ many $B_{\vec{k}}$ such that $B \cap B_{\vec{k}} \neq \emptyset$, and hence

$$
\Phi_{\alpha}\left(u_{m}, 2 B\right) \lesssim r_{B}^{2 \alpha-n} 2^{\ell n} 2^{-m(2-2 \alpha+n)} \lesssim 2^{2 \alpha \ell} 2^{-m(2-2 \alpha+n)} \lesssim 2^{-2 m},
$$


where $\ell=m(n-2 \alpha) / 2 \alpha$.

Finally, we have

$$
\frac{\left\|u_{m} \circ f\right\|_{Q_{\alpha}\left(\mathbb{R}^{n}\right)}}{\left\|u_{m}\right\|_{Q_{\alpha}\left(\mathbb{R}^{n}\right)}} \gtrsim 2^{m(n-2 \alpha) \beta /(\beta+1)} \rightarrow \infty
$$

as $m \rightarrow \infty$ since $\beta>0$.

Case 2: $\alpha_{0} \in(0,1)$. Similarly to Case 1: $\alpha_{0}=1$, we can first construct quasiconformal mappings $f: \mathbb{R}^{n} \rightarrow \mathbb{R}^{n}$ with $J_{f} \in A_{1}\left(\mathbb{R}^{n},\left(2^{\mathbb{N}_{\theta}}\right)^{n}\right)$, and then construct the critical function $u_{m}$ similarly to Case 1: $\alpha_{0}=1$, but the key parameter $\ell$ over there is now taken as $m(n-2 \alpha) /(2 \alpha-n+\theta n)$ where

$$
2 \alpha-n+\theta n>0 \Leftrightarrow 2 \alpha>n-\theta n=\alpha_{0} .
$$

Such a $\mathbf{C}_{f}$ is not bounded on $Q_{\alpha}\left(\mathbb{R}^{n}\right)$ for all $\alpha \in\left(\alpha_{0}, 1\right)$ and hence satisfies our requirement; we omit the details.

Acknowledgements. The third and forth authors would like to thank Professor Jacques Peyriere for kind discussions on the Minkowski type dimension. Part of this research was done during the first and forth authors' visit at IPAM, UCLA; both authors would like to thank for the support.

\section{REFERENCES}

[1] K. Astala, A remark on quasiconformal mappings and BMO-functions. Michigan Math. J. 30 (1983), $209 i ; \frac{1}{2} 212$.

[2] M. Bourdon and H. Pajot, Cohomologie $l_{p}$ et espaces de Besov. J. Reine Angew. Math. 558 (2003), 85-108.

[3] M. Essén, S. Janson, L. Peng and J. Xiao, $Q$ spaces of several real variables. Indiana Univ. Math. J. 49 (2000), 575-615.

[4] H. Koch, P. Koskela, E. Saksman and T. Soto, Bounded compositions on scaling invariant Besov spaces. arXiv:1209.6477.

[5] P. Koskela, Lectures on quasiconformal and quasisymmetrical mappings, University of Jyväskylä, 2011.

[6] P. Koskela, D. Yang and Y. Zhou, Pointwise characterizations of Besov and Triebel-Lizorkin spaces and quasiconformal mappings. Adv. Math. 226 (2011), 3579-3621.

[7] H. M. Reimann, Functions of bounded mean oscillation and quasiconformal mappings. Comment. Math. Helv. 49 (1974), 260-276.

[8] J. C. Robinson and W. Sadowski, On the dimension of the singular set of solutions to the Navier-Stokes equations. Commun. Math. Phys. 309 (2012), 497-506.

[9] P. Tukia and J. Väisälä, Quasiconformal extension from dimension $n$ to $n+1$. Ann. of Math. (2) 115 (1982), $331 i i \frac{1}{2} 348$.

[10] S. K. Vodop'janov, Mappings of homogeneous groups and embeddings of function spaces. Sib. Math. J. 30 (1989), 25-41.

Pekka Koskela: Department of Mathematics and Statistics, P.O. Box 35 (MaD), FI-40014, University of Jyväskylä, FINLAND

E-mail address: pkoskela@maths.jyu.fi

Jie Xiao: Department of Mathematics and Statistics, Memorial University, NL A1C 5S7, Canada

E-mail address: jxiao@mun.ca

Yi Ru-Ya Zhang: Department of Mathematics, Beijing University of Aeronautics and Astronautics, Beijing 100191, P.R. China and Department of Mathematics and Statistics, P.O. Box 35 (MaD), FI-40014, University of JyväSkylä, FINLAND

E-mail address: ddfx023zy@gmail.com 
Yuan Zhou: Department of Mathematics, Beijing University of Aeronautics and Astronautics, Beijing 100191, P.R. CHINA

E-mail address: yuanzhou@buaa.edu.cn 